ÉGYPTE monde arabe

\section{Égypte/Monde arabe}

$1 \mid 1990$

Modes d'urbanisation en Égypte

\title{
L'évolution des structures de l'emploi dans le centre du Caire (1976-1986)
}

Jacques Charmes

\section{OpenEdition}

\section{Journals}

Édition électronique

URL : https://journals.openedition.org/ema/979

DOI : 10.4000/ema.979

ISSN : 2090-7273

Éditeur

CEDEJ - Centre d'études et de documentation économiques juridiques et sociales

Édition imprimée

Date de publication : 31 mars 1990

Pagination : 125-151

ISSN : 1110-5097

\section{Référence électronique}

Jacques Charmes, «L'évolution des structures de l'emploi dans le centre du Caire (1976-1986) »,

Égypte/Monde arabe [En ligne], 1 | 1990, mis en ligne le 08 juillet 2008, consulté le 07 juillet 2022. URL: http://journals.openedition.org/ema/979; DOI : https://doi.org/10.4000/ema.979

Ce document a été généré automatiquement le 7 juillet 2022.

Tous droits réservés 


\title{
L'évolution des structures de l'emploi dans le centre du Caire (1976-1986)
}

\author{
Jacques Charmes
}

$1 \mathrm{Au}$ cours des dix années qui se sont écoulées entre les deux recensements de la population de 1976 et de 1986. la population active habitant le centre ville s'est accrue de près de 8,4\% (passant de 494324 à 536068 habitants), cependant que la population totale habitant le centre diminuait de $14,7 \%$ (passant de 1800000 à 1535000 habitants). Une part de la population active vivant au centre vide travaille en dehors de la zone: en 1976 et d'après le recensement. 18,5\% de ces actifs se déplaçaient quotidiennement pour travailler dans un autre quartier de la ville. On peut penser que, parmi ces actifs se déplaçant quotidiennement, un certain nombre de migrants ont définitivement quitté le centre ville, mais les résultats du recensement de la population de 1986 ne sont pas encore disponibles, qui pourraient nous fournir des données récentes et des indications sur les transformations intervenues en ce domaine au cours des 10 années intercensitaires.

2 En vue d'une meilleure compréhension du fonctionnement du marché du travail dans le centre du Caire, on se propose de dresser une typologie basée sur les caractéristiques structurelles et de comportement de la population active, des emplois et des établissements dans les différents qism du centre ville et leur évolution au cours de la période intercensitaire. Bien que la comparaison soit rendue difficile en raison des modifications des limites des qism, on fait l'hypothèse que ces changements n'affectent pas les principales caractéristiques de l'activité économique des qism et qu'une telle tentative de brosser une image structurelle et dynamique reste possible. Étant donné la provisoire, mais sans doute encore longue, non disponibilité des données par chiyakha, une telle hypothèse est indispensable. En tout état de cause, et à condition de ne pas accorder trop d'importance aux chiffres absolus et d'accorder plus de poids aux chiffres relatifs et aux données de structures, l'exercice peut s'avérer utile.

Sources et types de données disponibles au niveau des 
3 En 1976, ont été réalisés un recensement de la population et un recensement des établissements dont les résultats ont été publiés en 1981. Ces deux opérations ont été renouvelées en 1986, mais seuls des résultats préliminaires en sont disponibles sur la population active et sur l'emploi dans les établissements (sur le lieu de travail).

En 1976, il est possible de disposer de résultats détaillés par qism pour :

- La population par sexe et groupe d'âge,

- La population active, occupée et sans travail, par sexe,

- Les principales tranches d'activité économique,

- La répartition de la population active selon le lieu d'habitation et le lieu de travail,

- Le nombre d'établissements et d'emplois par branches d'activité économique,

- L'emploi selon la taille des établissements et l'activité économique.

En 1986, les résultats préliminaires fournissent, par qismJes tableaux suivants :

- La population par sexe et groupe d'âge,

- La population active, occupée et sans travail, par sexe,

- Le nombre d'établissements et d'emplois par sexe,

- Le nombre d'établissements selon le statut d'activité (en activité ou fermé),

- Le nombre d'établissements par secteur institutionnel (administration. public, privé),

- La répartition des logements selon le type d'utilisation (habitation, activité).

6 Sur la base de ces sources, nous présentons tout d'abord les données démographiques sur la population et la population active vivant au centre du Caire, avant d'étudier le niveau et la structure des emplois exercés au centre ville, et de proposer un bilan des échanges et des déplacements de population active entre le centre du Caire et le reste du gouvernorat.

Démographie du centre du Caire, population et population active vivant au CentreVillePopulation

7 Entre les deux recensements de 1976 et 1986, la population du centre ville, définie comme étant constituée des 15 qism énumérés sur le tableau 1 (voir en annexe les tableaux cités) a diminué de près de $15 \%$ (soit un taux annuel de $1.6 \%$ ) alors que dans le même temps, la population du gouvernorat du Caire augmentait de près de $19 \%$ (1,8\% par an). La population de tous les qism du centre ville a diminué à l'exception de Manchiyyat Nasser et de Zâmalek : encore ces exceptions peuvent-elles sans doute s'expliquer par les modifications des limites administratives de ces deux qism.

Population active et chômage

8 Le taux brut d'activité (ratio de la population active à la population totale) de la population du centre du Caire a augmenté de 31,3\% en 1976 à 34,9\% en 1986, mais le taux net d'activité (ratio de la population occupée à la population totale) a connu une croissance plus lente. En fait, les tendances qui viennent d'être indiquées sont dues à un très fort accroissement du taux de chômage (qui est passé de 7,8\% à 17,3\% entre 1976 et 1986 pour le centre du Caire aussi bien que pour le Gouvernorat du Caire). Cependant, l'une des principales raisons de cet accroissement est à rechercher dans les changements de définition du chômage et l'application par le CAPMAS (Central Agency for Public Mobilisation and Statistics) des normes internationales en matière de définition et de mesure du phénomène. La relative faiblesse des taux de chômage en 1976 s'explique en effet par la définition relativement floue du chômage et de l'activité : l'Egypte est alors le seul pays pour lequel la période de référence de l'enquête n'a pas été précisée, ce qui signifie que c'est en réalité l'activité habituelle qui a été relevée et 
qu'il suffit d'avoir travaillé même pour une courte durée dans l'année pour être considéré comme occupé.

On doit également considérer que le retournement de la situation économique depuis 1984 a été enregistré par le recensement de 1986, ce qui explique pourquoi le rapide accroissement de la population active, en réponse à une forte offre d'emplois après 1975 a été suivi d'une aggravation du taux de chômage aux alentours de 1986. La détérioration du marché du travail a affecté de façon contrastée la population vivant dans les qism du centre ville, comme le montre le tableau 2.

En 1976, trois qism seulement ont connu des taux de chômage supérieurs à la moyenne : Muski (11,4\%), Sayyida Zaynab (11.3\%) et Zahir (9,0\%). En 1986, Darb al-Ahmar dont la population active a légèrement augmenté, connaît un taux de chômage très élevé (38,0\%). probablement le plus élevé du Caire; Azbakiyya (22,7\%), 'Abdin (21,1\%) et Bab al-Sharia $(21,1 \%)$ sont également des quartiers qui se caractérisent par des hauts niveaux de chômage, suivis par Zahir (19,5\%) et al-Wayli (19,0\%).

11 Le critère du taux de chômage permet de classer les qism du centre ville en 5 catégories, comme l'indique le tableau 3. Etant donné la grande hétérogénéité de ces groupes, l'introduction d'autres critères dans l'analyse (comme l'importance des populations qui se déplacent pour travailler) est nécessaire pour expliquer les différences entre qism d'un même groupe.

Population d'âge actif

Entre 1976 et 1986, la répartition de la population d'âge actif par qism n'a guère changé (voir tableau 4). Dans le centre ville, la population d'âge actif (entre 12 et 65 ans... On notera cependant que le critère d'âge utilisé par le CAPMAS pour définir la population active est de 6 ans ou plus) a légèrement augmenté, passant de $71.2 \%$ à $72,2 \%$, et elle a légèrement diminué dans l'ensemble du gouvernorat du Caire, passant de $70,5 \%$ à $68,1 \%$. Au centre ville, 2 catégories de qism peuvent être distinguées :

- Une première catégorie se caractérise par une forte proportion de population d'âge actif (76 à $78 \%$ en 1976 aussi bien qu'en 1986) : Zahir, 'Abdin, Azbakiyya et Qasr el-Nil, Zamalek.

- Une seconde catégorie où la proportion de population d'âge actif est relativement faible (de 60 à 70\%) : Manchiyyat Nasser, Masr al-Qadima et Khalifa. Il est intéressant de noter que parmi les qism caractérisés par une forte proportion de population d'âge actif, une première sous-catégorie a connu des taux de chômage élevés en 1976 et 1986 (Zahir, 'Abdin et Azbakiyya), tandis qu'une seconde sous-catégorie se caractérise par de bas niveaux de chômage (Qasr al-Nil, Zamalek). Dans ces deux derniers qism, les habitants sont plus âgés et donc moins affectés par le chômage (les habitants de plus de 65 ans y représentent respectivement 8,3 et $6,8 \%$ de la population) alors qu'à Manchiyyat Nasser, les jeunes (de moins de 12 ans) représentent $39,7 \%$ de la population. De plus, l'emploi dans ces qism est étroitement lié à des activités tertiaires de haut niveau qui n'ont pas été affectées par la crise économique actuelle.

Profession et statuts dans la profession La répartition de la population active vivant dans les qism du centre ville selon la profession et le statut dans la profession (employeurs, indépendants, aides familiaux, salariés) est mieux à même de nous donner un éclairage intéressant sur le statut social de cette population que la répartition par branche d'activité économique. Les tableaux 5 et 6 , et surtout le tableau 7 qui les résume, montrent que la population vivant à Qasr al-Nil, Zahir, al-Wayli et 'Abdin appartient à une catégorie sociale élevée 
de « cols blancs» (professions techniques, administratives et d'encadrement) dans une proportion allant de $42,5 \%$ pour Qasr al-Nil à $21,8 \%$ pour 'Abdin, alors que les catégories sociales de cols blancs moins élevées (employés) vivent à Zahir, Sayyida Zaynab, 'Abdin et al-Wayli. Au contraire, les qism où les ouvriers du commerce, des services et de la production prédominent sont al-Gamaliyya, Bulaq, Muski et Bab al-Sharia (de 78,3 à $70,1 \%$ de la population active) et dans une moindre mesure, Khalifa, Darb al-Ahmar, Azbakiyya présentent des résultats plus contrastés, assez proches des moyennes pour le gouvernerai du Caire dans son ensemble. Cette répartition des qism dans le centre ville est confirmée lorsqu'on utilise le critère du statut dans la profession, les indépendants (auto emploi) et les travailleurs non rémunérés (aides familiaux et apprentis) sont relativement plus nombreux dans ces mêmes qism où prédominent les ouvriers.

Les déplacements vers les lieux de travail

Pour l'année 1976, les résultats du recensement de la population fournissent un intéressant ensemble de données sur la répartition de la population active, selon le lieu d'habitation et le lieu de travail. Ces données ne sont pas encore disponibles pour 1986. On considérera cependant que les résultats de 1976 sont suffisamment intéressants pour être présentés comme éléments d'explication de la situation présente.

Cependant, ces résultats ne concernent que le gouvernorat du Caire et ne fournissent aucune indication sur les déplacements qui s'effectuent entre ce gouvernorat et ceux de Giza et Qalyubîya. Quelques caractéristiques intéressantes sur la population active et l'emploi peuvent néanmoins être tirées des données présentées aux tableaux 8 et 9. Le tableau 8 est un essai d'agrégation des données du CAPMAS répartissant la population du Gouvernorat du Caire selon le qism d'habitation et selon 4 catégories de lieu de travail :

- Le qism d'habitation dans le centre ville,

- Le quartier central des affaires (Central Business District CBD), grossièrement défini comme constitué par les 3 qism de Qasr al-Nil, 'Abdin, Azbakiyya),

- Le centre ville (les 13 qism étudiés, devenus 15 en 1986).

- Le reste du gouvernorat du Caire (à l'exception du centre ville). Où travaillent les habitants d'un qism déterminé ? La matrice lieu d'habitation/lieu de travail permet de répondre a cette question (voir tableau 9).

Les qism qui retiennent le plus leur population sont ceux de Qasr al-Nil (où $71 \%$ de la population travaille sur place), Azbakiyya (59\%) et Bulaq (59\%), tandis que les qism qui la retiennent le moins sont ceux de Muski, Zahir (35\%) et Khalifa (44\%). Si l'on excepte les qism qui appartiennent au centre des affaires (CBD Qasr al-Nil, Azbakiyya et 'Abdin), la matrice montre que les autres qism du centre ville envoient travailler dans le centre des affaires une faible proportion de leur population (de 8 à $9 \%$ pour al-Gamaliyya et Muski, à 20,23\% pour Sayyida Zaynab et Zahir). Cependant, parmi les 196234 emplois occupés dans le centre des affaires par les habitants du gouvernorat du Caire, il y en a $38042(19,4 \%)$ qui sont tenus par des habitants du centre des affaires, 64170 (32,7\%) par les habitants du reste du centre ville et $94022(47,9 \%)$ par des personnes vivant dans d'autres qism du gouvernorat. On ne peut malheureusement pas dire, à partir de ces données, combien d'habitants de Giza viennent travailler chaque jour dans le centre des affaires.

Les habitants du centre du Caire travaillent au centre ville dans une proportion qui varie de $77 \%$ pour al-Gamaliyya (mais dans ce qism, la catégorie «indéterminés » est très nombreuse et est susceptible de provoquer une importante distorsion) à $91 \%$ pour 
Qasr al-Nil-. Muski est une exception avec une faible proportion de 53\% : dans ce qism, $47 \%$ des habitants se déplacent chaque jour hors du centre ville pour travailler, alors que cette proportion n'est que de $15 \%$ pour les habitants du centre ville dans leur ensemble. Cette particularité de Muski doit être soulignée et mériterait d'être expliquée.

De même, la catégorie "indéterminés » est d'une interprétation difficile, puisqu'elle comprend des réponses incorrectes ou incomplètes, aussi bien que les réponses de ceux qui se déplacent hors du Gouvernorat du Caire. Le tableau 9 présente la matrice lieu de travail/lieu d'habitation. Il doit être lu de la façon suivante : combien de personnes travaillant dans un qism déterminé (donné en ligne sur le tableau) vivent dans un quartier déterminé (donné en colonne). Le tableau révèle que les qism où l'on observe les plus fortes proportions de personnes vivant et travaillant à l'intérieur même du qism sont aussi ceux qui drainent la plus forte proportion de travailleurs vivant à l'extérieur : en d'autres termes, ils constituent des pôles d'emploi. Le centre des affaires est un tel pôle, orienté vers les activités tertiaires, avec Qasr al-Nil, Azbakiyya et, dans une moindre mesure, 'Abdin, où les habitants ne détiennent respectivement que 10,16 et $27 \%$ des emplois localisés dans la zone. Un autre pôle à base d'activités manufacturières, est al-Wayli, où $26 \%$ seulement des emplois sont occupés par des habitants du qism et $54 \%$ des actifs travaillant dans les activités localisées dans la zone proviennent de l'extérieur du centre ville. L'examen de ces deux matrices permet, en conclusion, de souligner qu'en 1976, 15\% seulement des habitants du gouvernoray du Caire quittaient quotidiennement le centre ville, et $24 \%$ s'y rendaient pour leur travail.

Il est maintenant nécessaire de déterminer le nombre et la répartition des emplois effectivement localisés au centre ville. Cela pourra nous donner la mesure des déplacements vers le centre ville en provenance des autres gouvernorats. Une telle approche doit se fonder sur les données d'emploi, en particulier les résultats du recensement des établissements.

Niveau et structures de l'emploi dans le centre du Caire

Les principales sources permettant une localisation précise des emplois dans le centre du Caire sont les recensements d'établissements. Des résultats sont disponibles pour les années 1972, 1976 et 1986 (résultats préliminaires) Mais, lorsqu'on analyse ces résultats et en particulier lorsqu'on les compare avec les données de population active, présentées au paragraphe précédent, on doit garder à l'esprit le fait qu'ils n'incluent pas les emplois de l'administration et des services publics).

L'emploi dans les établissements

Du tableau 10, il ressort que le nombre d'établissements dans le centre du Caire a augmenté de près de $14 \%$ en 4 ans, entre 1972 et 1976, puis de $12 \%$ seulement dans les 10 années qui séparent 1976 et 1986 . Au cours des mêmes périodes, l'emploi dans les établissements a augmenté de 5,4\% entre J972 et 1976, mais a légèrement diminué de près de 2,6\% entre 1976 et 1986.

Si l'on se concentre maintenant sur la période 1976-86, le tableau 11 permet de comparer les taux de croissance du nombre d'établissements et du nombre d'emplois. Les diminutions d'emplois les plus importantes ont été enregistrées à 'Abdin (-29,9\%), al-Wayli $(-27,5 \%)$. Zahir (-18\%), Sayyida Zaynab (-16,9\%) et Azbakiyya (-9,7\%). Au contraire, Qasr al-Nil (y compris Zamalek) a connu un taux de croissance de l'emploi très élevé $(36,8 \%)$ qui ne revient cependant pas à compenser les pertes à 'Abdin. La croissance de l'emploi à Khalifa a également été importante (16\%). Quant à al- 
Gamaliyya et Darb al-Ahmar, leur niveau d'emploi est resté stable, bien que ces deux qism aient perdu une partie significative de leur superficie et de leur population qui constitue aujourd'hui le qism de Manchiyyat Nasser; mais si nous ajoutions aux effectifs de ces 2 qism le nombre d'emplois à Manchiyyat Nasser, la croissance de remploi s'élèverait alors à $15.3 \%$.

Le nombre d'établissements par habitat est un ratio qui rend les données de 1976 et de 1986 plus comparables, étant donné les changements intervenus dans les limites des qism. Le tabteau 12 montre que ce ratio a augmenté dans tous les qism. A Zahir, Azbakiyya et 'Abdin, où le nombre d'établissements a diminué, la croissance du ratio signifie donc que la population a décru encore plus rapidement. Le fort accroissement du ratio à al-Gamaliyya $(+100 \%)$ mérite d'être relevé, mais il peut être en partie dû aux nouvelles limites du qism qui le restreignent aux zones de fortes densités d'activités. Muski et Qasr al-Nil-Zamalek sont des qism où le ratio, déjà élevé en 1976, a continué à croître fortement (de 50 et $40 \%$ respectivement).

L'emploi par branche d'activité économique :

Quels sont, selon les données statistiques disponibles, les divers types d'activités dans lesquels sont spécialisés les différents qism, et le centre ville dans son ensemble?

La répartition des emplois par branche et secteur d'activité n'est disponible que pour la seule année 1976, à partir des résultats du recensement des établissements. Comme le montre le tableau 13, les activités manufacturières fournissaient plus de $34 \%$ des emplois non administratifs du centre du Caire en 1976; le commerce y contribuait pour sa part à raison de $30 \%$, et les services pour $30 \%$ également.

Cette répartition équilibrée est la résultante de distributions différentes au niveau des qism. Les qism à dominante manufacturière sont Bab al-Charia (avec 53\% des emplois dans ce secteur), al-Gamaliyya (50\%), Khalifa (46\%), Muski et Bulaq (45\%). Mais, au niveau du centre du Caire, 'Abdin et al-Gamaliyya sont les deux qism qui procurent le plus grand nombre d'emplois manufacturiers (13 et $12 \%$ respectivement). AlGamaliyya, Darb al-Ahmar et Muski se caractérisent par une forte proportion d'emplois dans le commerce (près de 40\%), mais ce sont 'Abdin (18\%), Azbakiyya (13\%) et alGamaliyya (11\%) qui contribuent le plus à l'emploi commercial du centre ville. En ce qui concerne les services, 'Abdin (49\%), Zahir (41\%), Masr al-Qadima (32\%) et Qasr al-Nil (37\%) sont les qism qui peuvent être considérés comme les plus spécialisés dans ces activités. 'Abdin à lui seul contribue pour plus de $36 \%$ à l'emploi total dans les services du centre ville, et pour près de $21 \%$ à l'échelle du gouvernorat tout entier.

Si nous nous intéressons maintenant aux divers types d'industries manufacturières (Tableau 14), il apparaît que al-Gamaliyya, 'Abdin et Muski sont les principaux fournisseurs d'emplois dans le textile, l'habillement et le cuir, avec respectivement 17, 17 et $15 \%$ de l'emploi de ce secteur dans le centre ville (qui concerne lui-même près de $50 \%$ de ces emplois à l'échelle du gouvernorat). C'est dans ces mêmes qism que remploi dans le textile, rhabillement et le cuir représente la plus forte proportion de l'emploi manufacturier (56\% à Muski, $46 \%$ à 'Abdin et $45 \%$ à al-Gamaliyya). Mais le textile est aussi une spécialisation pour Masr at-Qadima et Khalifa (avec 46\% de leur emploi manufacturier). Al-Wayti, Bulaq et al-Gamatiyya sont des qism spécialisés dans les industries métalliques (avec respectivement 35.28 et $25 \%$ de leur emploi manufacturier dans cette seule activité) et les industries du bois et de l'imprimerie : Bulaq (51\%), Darb al-Ahmar (42\%) et Zahir (38\%). Alors que al-Gamaliyya et Bulaq contribuaient pour 16,5 et $15,7 \%$ de l'emploi des industries métalliques dans le centre ville, cette proportion 
était de $12 \%$ pour al-Wayli et 'Abdin. Dans les industries du bois et de l'imprimerie, Bulaq a un poids très élevé (21\%), suivi par Darb al-Ahmar (13\%), Sayyida Zaynab (11\%) et Azbakiyya (10\%).

Pour les trois principales, industries manufacturières, il est intéressant de retracer les résultats sur les graphiques du tableau 15. La taille des établissements est une autre caractéristique intéressante pour différencier les qism du centre du Caire. On a déjà vu que la taille moyenne des établissements avait diminué de 1976 à 1986. Selon le tabteau 10, la taille moyenne des établissements du centre ville était en 1976 légèrement inférieure $(4,12)$ à celle qu'elle était dans l'ensemble du gouvernorat $(4,22)$. Cela signifie que les grandes entreprises avaient plutôt tendance à s'installer à la périphérie, hors du Caire. C'est la situation inverse que l'on observe en 1986 (3,59 contre 3,56), à un niveau plus bas.

30 Le tableau 16 montre que les qism où la taille moyenne (les établissements est la plus faible sont Khalifa (2,27), Darb af-Ahmar (2,36), Bab al-Charia (2.67), Sayyida Zaynab $(2,75)$ et al-Gamaliyya (2.80). Les qism où cette taille est la plus élevée sont ceux du centre des affaires Qasr al-Nil (10.01), 'Abdin (7,09). Al-Azbakiyya $(5,27)$ et al-Wayli $(4,51$ ), la taille moyenne est également assez élevée.

31 La taille moyenne des établissements est faible dans les activités commerciales $(2,65)$, les services personnels $(2,87)$ et les restaurants $(3,32)$; elle est élevée dans les transports $(18,7)$, les banques $(14,74)$ et la construction $(13,49)$. Dans les industries manufacturières, la taille moyenne est plutôt faible (4.03), mais le tableau 19 montre qu'il y a un nombre appréciable de grandes entreprises manufacturières à af-Wayli, Bulaq, Qasr al-Nil. Azbakiyya et Masr al-Qadima. De la même façon, les grandes entreprises du commerce se concentrent dans le centre des affaires.

Les activités dans les locaux à usage d'habitation

Une autre caractéristique intéressante des différents qism du centre ville est la proportion des unités d'habitations utilisées pour l'exercice d'une activité : ce ratio caractérise la façon dont les activités s'étendent dans le centre du Caire.

Le tableau 17 présente les résultats des recensements de la population de 1976 et 1986. Précédant immédiatement les opérations censitaires, un dénombrement des locaux dotation a été effectué, qui permet (le distinguer ceux qui sont exclusivement destinés à l'usage d'habitation de ceux qui sont utilisés pour l'exercice d'une activité économique (une catégorie à usage mixte est incluse dans ce dernier type).

Ce ratio est particulièrement élevé (plus de 2 fois supérieur à la moyenne du centre ville) à 'Abdin ( $22,2 \%$ en 1976 et $22,8 \%$ en 1986), Azbakiyya (18,5; 20.2), Muski $(14.6 ; 19,5)$ et Qasr al-Nil $(11.8 ; 15.4)$ où les bureaux et les diverses activités tertiaires en grand nombre expliquent largement ce phénomène. Dans les qism où les activités manufacturières et informelles sont plus nombreuses, le ratio est beaucoup plus faible, sauf à Darb al-Ahmar et al-Gamaliyya (proches de la moyenne du centre ville). Il a fortement décru à Darb al-Ahmar en 1986, sans doute à cause des nouvelles limites du qism.

Le tableau 18 montre l'évolution du ratio pour les différents qism entre 1976 et 1986, en mettant l'accent sur les taux de croissance ou de décroissance les plus élevés. Les recensements d'établissements donnent des résultats différents en ce qui concerne la proportion d'activités exercées dans des logements, alors que les recensements de population dénombrent les locaux à usage d'habitation et demandent quel type d'usage 
en est fait, les recensements d'établissements dénombrent les activités et demandent (ou plutôt observent) si l'activité est exercée dans un logement, il peut donc exister un écart entre les deux sources : nous appellerons cet écart le «nombre d'établissements cachés" et nous cherchons à savoir si l'importance de ces établissements cachés explique ou non les différences observées aux tableaux 10,11 et 12 .

Le tableau 19 présente un essai d'estimation du nombre d'établissements cachés. Il est fondé sur l'hypothèse selon laquelle, en 1986, la proportion des établissements situés dans des logements et dénombrés par le recensement des établissements est restée au même niveau qu'en 1976 : cela signifie que le ratio de la colonne 3 (1976) a été appliqué aux effectifs de la colonne 1 (1986) en vue de calculer les effectifs de la colonne 8 (1986). L'examen des données et des résultats du tableau précédent nous montre que le nombre d'établissements recensés dans le centre du Caire a été sous-estime de quelques 9321 unités $(8.9 \%)$ en 1986. Sur la base d'une hypothèse de taille moyenne par établissement de 2.5 à 3 emplois, le niveau réel de l'emploi au centre ville s'élèverait à près de 405000 emplois (sans compter l'administration ni les services publics. Ces résultats et ces hypothèses nous permettent de proposer un bilan de l'emploi dans le centre du Caire et de mieux approcher les échanges et les déplacements de population qui s'opèrent entre le centre ville et le reste du Gouvernorat.

Bilan de l'emploi dans le centre du Caire et essai d'estimation des échanges de population entre le centre-ville et le reste du gouvernorat du Caire

La population active occupée vivant au centre du Caire s'élève en 1986 à 443500 personnes (et tableau 2). Les matrices lieu d'habitation/lieu de travail nous ont permis de savoir quelle était, en 1976, la proportion de population active vivant dans le centre ville qui se déplaçait quotidiennement vers les quartiers périphériques afin d'exercer une activité économique; de même, ces matrices permettent de savoir quelle est la proportion des actifs du gouvernorat qui se rend quotidiennement au travail dans le centre ville. Selon le tableau 8, 71971 personnes se déplaçaient pour leur travail hors du centre ville et à l'intérieur du gouvernorat, auxquelles s'ajoutent quelques 19454 indéterminés dont on peut penser qu'ils sont des actifs se déplaçant vers les autres gouvernorats (codification non prévue dans l'exploitation de la question); en tout, 91 426 personnes quittaient quotidiennement le centre ville pour aller travailler ailleurs, soit 17.6\% de la population occupée vivant au centre ville en 1976 (91 426 / 518 845). Le tableau 9 nous indique par ailleurs que 209915 actifs vivant dans le gouvernerai du Caire (et hors du centre ville) se rendaient quotidiennement au centre pour exercer leur activité, soit $24,0 \%$ de la population active du gouvernerai, hors centre ville (209 915 /1 394 025-518 845).

39 Le bilan de l'emploi au centre ville s'établit donc comme suit :

-1. Population active occupée du gouvernorat du Caire : 1552300

-2. Population active occupée (vivant au centre ville) : 443500

-3. moins les actifs se déplaçant hors du centre ville : 17,6\% de (2) -78 100

-4. plus les actifs du reste du gouvernerai venant au centre ville : $24 \%$ de (1) - (2)) + 266100

-5. nombre d'emplois détenus au centre ville par des habitants du gouvernorat : 631500.

Ce chiffre est néanmoins incomplet, car il conviendrait d'y ajouter les habitants du gouvernerai de Giza qui viennent travailler au centre ville. Par ailleurs, on sait que les 104869 établissements recensés au centre ville en 1986 empiètent 376519 personnes. Et l'on a estimé à 9321 le nombre d'établissements cachés qui emploieraient quelque 26 600 personnes. Au total, près de 402200 emplois peuvent ainsi être localisés au centre 
ville. A ce chiffre, il conviendrait d'ajouter les effectifs employés par l'Administration et les services publics, ainsi que tous les petits métiers exercés dans les rues de façon itinérante ou clandestine. En définitive, trois informations continuent à manquer en vue de parvenir à l'équilibre de l'emploi au centre du Caire : statistique et quantitative ne peut dispenser le chercheur et le planificateur urbain d'entreprendre une démarche qualitative en vue de connaître les problèmes des habitants et des travailleurs du centre du Caire, notamment lorsqu'il s'agit de définir et d'élaborer des projets visant à déconcentrer certaines activités économiques et à convaincre les habitants de certains quartiers de se déplacer vers de nouveaux sites d'habitat.

\section{ANNEXES}

Tableau 1 : Population du Centre du Caire par OIsm, en 1976 et en 1986 


\begin{tabular}{|l|r|r|c|}
\hline \multicolumn{1}{|c|}{ QISMS } & $\begin{array}{c}\text { Population } \\
1976\end{array}$ & $\begin{array}{r}\text { Population } \\
1986\end{array}$ & $\begin{array}{c}\text { Taux annuel } \\
\text { de croissance }\end{array}$ \\
\hline al-Wayli & 141,849 & 112,596 & $-2,3 \%$ \\
Manchiyyat Nasser (1) & 55,955 & 130,240 & $8,8 \%$ \\
Zahir & 104,354 & 83,816 & $-2,2 \%$ \\
Bab al-Charia & 110,293 & 79,562 & $-3,2 \%$ \\
al-Gamafiya (1) & 123,842 & 89,841 & $-3,2 \%$ \\
Darb al-Ahmar (1) & 133,404 & 105,208 & $-2,3 \%$ \\
Azbakiyya & 59,473 & 45,373 & $-2,7 \%$ \\
Muski & 58,189 & 43,201 & $-2,9 \%$ \\
Bulaq & 177,476 & 123,376 & $-3,6 \%$ \\
Abdin & 87,759 & 65,090 & $-2,9 \%$ \\
Zamalek (1) & 18,784 & 21,716 & $1,5 \%$ \\
Qasr al-Nil (1) & 19,532 & 17,204 & $-1,3 \%$ \\
Sayyida Zaynab & 252,090 & 198,838 & $-2,3 \%$ \\
Khalifa & 186,359 & 163,897 & $-1,3 \%$ \\
Masr al-Oadima & 270,343 & 254,651 & $-0,6 \%$ \\
\hline Total Centre Ville & 1799702 & 1534609 & $-1,6 \%$ \\
\hline Total Le Caire & 5074016 & 6052836 & $1,8 \%$ \\
\hline
\end{tabular}

(1) Nouvelles limites administratives en 1986

Sources : CAPMAS, Recensements de population 1976,1986

Tableau 2 : Répartition de la population par qism en 1976 et 1986

\begin{tabular}{|c|c|c|c|c|c|c|c|c|c|c|}
\hline \multirow[b]{2}{*}{ QISMS } & \multicolumn{5}{|c|}{1976} & \multicolumn{5}{|c|}{1988} \\
\hline & $\begin{array}{c}\text { Population } \\
\text { occup a }\end{array} \mid$ & $\begin{array}{l}\text { Population } \\
\text { sans travail }\end{array}$ & $\begin{array}{l}\text { Taux de } \\
\text { chomage }\end{array}$ & $\begin{array}{l}\text { Population } \\
\text { active }\end{array}$ & $\begin{array}{c}\text { Taux } \\
\text { d'activites }\end{array}$ & $\begin{array}{c}\text { Population } \\
\text { occupae }\end{array} \mid$ & $\begin{array}{l}\text { Population } \\
\text { sans travail }\end{array}$ & $\begin{array}{l}\text { Taux de } \\
\text { chomage }\end{array}$ & $\begin{array}{c}\text { Population } \\
\text { active }\end{array}$ & $\begin{array}{c}\text { Taux } \\
\text { d'activite }\end{array}$ \\
\hline $\begin{array}{l}\text { A) Wayil } \\
\text { Manchiyyat Nasser }\end{array}$ & 50510 & 4137 & $7.6 \%$ & 54647 & $38,5 \%$ & $\begin{array}{l}32272 \\
34461\end{array}$ & $\begin{array}{l}7590 \\
2197\end{array}$ & $\begin{array}{r}19.0 \% \\
6.0 \%\end{array}$ & $\begin{array}{l}38862 \\
36658\end{array}$ & $\begin{array}{l}35,4 \% \\
28,1 \%\end{array}$ \\
\hline Zahir & 28894 & 2866 & $9,0 \%$ & 31760 & $30.4 \%$ & 23854 & 5796 & $19.5 \%$ & 29650 & $\begin{array}{l}28,18 \\
35,48\end{array}$ \\
\hline Bab al-Charia & 32894 & 2251 & $6,4 \%$ & 35145 & $31,9 \%$ & 23039 & 6181 & $21,2 \%$ & 29220 & $36,7 x$ \\
\hline al. Gamaliayya & 36112 & $\begin{array}{l}2931 \\
2931\end{array}$ & $7,5 \%$ & 39043 & $23.4 \%$ & 24868 & 3505 & $12,4 \%$ & 28373 & $31.6 \%$ \\
\hline Darb al-Ahmar & 43840 & 3840 & $8,1 \%$ & 47680 & $32.6 \%$ & 30399 & 18660 & $38,0 \%$ & 49059 & $46.6 \%$ \\
\hline Azbakiyya & 18714 & 1677 & $8.2 \%$ & 20391 & $34.3 \%$ & 13971 & 4102 & $22.7 x$ & 18073 & $39.8 \%$ \\
\hline Muski & 16836 & 2169 & $11.4 \%$ & 19005 & $32,7 \%$ & 12858 & 1375 & $9.7 \%$ & 14233 & $32,9 \%$ \\
\hline Bulaq & 51183 & 4234 & $7.6 \%$ & $\mathbf{5 5 4 1 7}$ & $31.2 \%$ & 38238 & 3717 & $8.9 \%$ & 41955 & $34,0 \%$ \\
\hline Abdin & 25378 & 2302 & $8.3 \%$ & 27680 & $31.5 \%$ & 19961 & 5341 & 21.18 & 23302 & $38,9 \%$ \\
\hline Cast al-Nil & 13397 & 809 & $5.7 \%$ & 14206 & $37.1 \%$ & 5802 & 534 & $8.4 \%$ & 6336 & $36.8 \%$ \\
\hline Zamalek & & & & & & 8008 & 387 & $4.6 \%$ & 8395 & $38.7 \%$ \\
\hline Sayyida Zaynab & 73445 & 6818 & $8,5 \%$ & 80263 & $31.8 \%$ & 59079 & 10586 & $15,2 x$ & 69665 & $35,0 \%$ \\
\hline $\begin{array}{l}\text { Khalita } \\
\text { Kaglo }\end{array}$ & 52309 & 4122 & $7.3 x$ & 56431 & $30.3 \%$ & 48291. & 10881 & $18.4 \%$ & 59172 & $36,1 \%$ \\
\hline Masr al-Oadima & 75333 & 5824 & $7,2 x$ & 81157 & $30,0 \%$ & 68405 & 11710 & $14.6 \%$ & 80115 & $31,5 \%$ \\
\hline Total Centre ville & 518845 & 43980 & $7.8 x$ & 562825 & $31,3 \%$ & 443506 & 92562 & $17,3 \%$ & 533068 & $34.9 \%$ \\
\hline Total Le Caire & 1394025 & 120101 & $7.9 \%$ & 1514126 & $29.8 \%$ & 1552287 & 324054 & $17.3 \%$ & 1876341 & $31.0 \%$ \\
\hline
\end{tabular}

Source : CAPMAS, Recensements de la population 1976, 1986

Taux de chômage : Population sans travail / Population active

Taux brut de la population active / Population totale

Tableau 3 : Typologie des qisms en 1976 et 1986 


\begin{tabular}{|c|l|l|l|}
\hline $\begin{array}{c}\text { Taux de chómage } 1986 \\
1976\end{array}$ & \multicolumn{1}{|c|}{ Faible } & Moyenne & \multicolumn{1}{c|}{ Forte } \\
\hline Faible & $\begin{array}{l}\text { Qasr al-Nil } \\
\text { Zamalek } \\
\text { al-Gamallyya }\end{array}$ & & $\begin{array}{l}\text { al-Wayli } \\
\text { Bab al-Charia }\end{array}$ \\
Moyenne & $\begin{array}{l}\text { Kalifa } \\
\text { Masr al-Oadima }\end{array}$ & \\
Elevé & $\begin{array}{l}\text { Muski } \\
\text { Sayyida Zaynab }\end{array}$ & & $\begin{array}{l}\text { Zahir } \\
\text { 'Abdin } \\
\text { Azbakiyya } \\
\text { Darb al-Ahmar }\end{array}$ \\
\hline
\end{tabular}

Tableau 4 : Population d'âge actif par qism 1976-1986

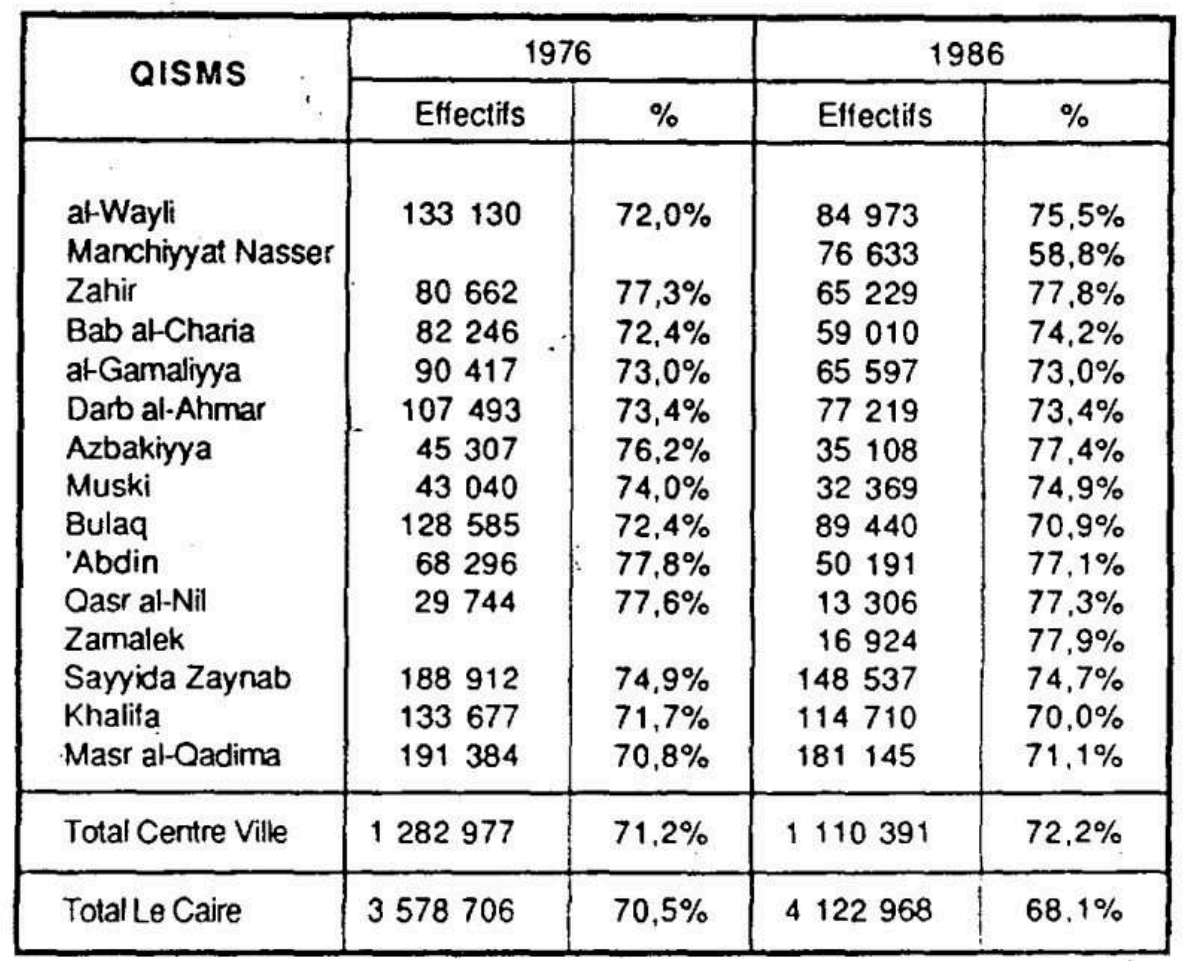

Source : Recensements de Population

Tableau 5 : Répartition de la population active selon la profession (15 ans au plus) 1976 


\begin{tabular}{|c|c|c|c|c|c|c|c|c|c|c|c|c|c|c|c|c|}
\hline \multirow{2}{*}{ aisms } & \multicolumn{16}{|c|}{ PROFESSION } \\
\hline & $\begin{array}{l}\text { Prolessions } \\
\text { scientifiques } \\
\text { ot lechniques }\end{array}$ & \multicolumn{2}{|c|}{$\begin{array}{c}\text { Cadres } \\
\text {.administratits }\end{array}$} & \multicolumn{2}{|c|}{ - Employés } & \multicolumn{2}{|c|}{$\begin{array}{l}\text { Personnel } \\
\text { commercial }\end{array}$} & \multicolumn{2}{|c|}{$\begin{array}{c}\text { Personnel } \\
\text { des } \\
\text { services }\end{array}$} & \multicolumn{2}{|c|}{\begin{tabular}{|c|} 
Travailleurs \\
de \\
ragriculture
\end{tabular}} & \begin{tabular}{|c|} 
Travailleurs \\
oo la production \\
des transports
\end{tabular} & \multicolumn{2}{|c|}{ Non classes } & \multicolumn{2}{|c|}{ Total } \\
\hline $\begin{array}{l}\text { A1. Wayli } \\
\text { Manchiyyat Nases }\end{array}$ & $7730 \quad 18,8 \%$ & & $3.4 \%$ & & $17.1 \%$ & 3549 & $8.6 \%$ & 5401 & $13,2 \%$ & 219 & $0,5 \%$ & $1211729.5 \%$ & 3622 & & 41053 & \\
\hline $\begin{array}{l}\text { Zahit } \\
\text { Bab Charia }\end{array}$ & $7010 \quad 22.5 \%$ & 1370 & $4.4 \%$ & 8104 & $19.6 \%$ & 3541 & $11,4 \%$ & 3059 & $9,8 \%$ & 67 & $0.2 \%$ & $6989 \quad 22.5 \%$ & 2971 & $9.5 \%$ & 31111 & 100, \\
\hline al & $3177 \quad 9.4 \%$ & & & & $13.0 \%$ & 4508 & & & $10.7 \%$ & 82 & & 1551 & & & & \\
\hline Darb & 2857 & 345 & & & $9,0 \%$ & 9255 & $19.0 \%$ & 5928 & $12,2 \%$ & 255 & $0.5 \%$ & $2301447.3 \%$ & 28 & $5,9 \%$ & 48770 & \\
\hline (a) & 4061 & 548 & $1,2 \%$ & & $12.3 \%$ & 6698 & $15.1 \%$ & 4604 & $10.4 \%$ & 1423 & $3,2 \%$ & 17917 & & $8,3 \%$ & 44394 & \\
\hline & 3212 & $\begin{array}{l}753 \\
252\end{array}$ & & & $14,3 \%$ & 3195 & & 2804 & $13,5 \%$ & 501 & $2,4 \%$ & $26,7 \%$ & 1824 & $8,8 \%$ & 20837 & \\
\hline & 1704 & 252 & & & $10,5 \%$ & 3598 & $20.1 \%$ & 1922 & $10,7 \%$ & 228 & $1,3 \%$ & $10,3 \%$ & 1107 & & & \\
\hline & $3172 \quad 6.0 \%$ & 494 & $0,9 \%$ & & $9.5 * 6$ & 8483 & $16,0 \%$ & 6840 & $13.0 \%$ & 125 & $0.2 \%$ & $24989 \quad 47.3 \%$ & 3718 & $7.0 \%$ & 52804 & \\
\hline & $504917.8 \%$ & 1136 & $4,0 \%$ & 5168 & $18,3 \%$ & 2913 & $10.3 \%$ & & $13,2 \%$ & & $0.3 \% 4$ & $7478 \quad 26,4 \%$ & & & & \\
\hline $\begin{array}{l}\text { Zast } \\
\text { Zannalich }\end{array}$ & $420330.8 \%$ & 1602 & $11.7 \%$ & 1578 & $11,6 \%$ & 1188 & $8.7 \%$ & 2940 & $21,5 \%$ & 139 & $1.0 \%$ & $928 \quad 6,1 \%$ & 1188 & $8.7 \times 6$ & 13682 & $100.0 \%$ \\
\hline $\begin{array}{l}\text { Saryida Zaynab } \\
\text { Knalita }\end{array}$ & $11974 \quad 15.4 \%$ & 2288 & $2.8 \%$ & 14516 & $18.7 \%$ & 7422 & & 9839 & & 357 & $0,5 \%$ & $2419331,2 \%$ & 6944 & $9.0 \%$ & & \\
\hline Mast al-Oadima & $\begin{array}{rr}5241 & 9,7 \% \\
12480 & 15,9 \%\end{array}$ & 2859 & $\begin{array}{ll}1,5 \% \\
3.8 \%\end{array}$ & 7463 & $\begin{array}{l}13,8 \% \\
14,1 \%\end{array}$ & $\begin{array}{l}4380 \\
7811\end{array}$ & $\begin{array}{l}8.1 \% \\
10.0 \% \\
8\end{array}$ & $\begin{array}{l}6455 \\
9785\end{array}$ & $12.0 \%$ & & $0.3 \%$ & $25529 \quad 47,3 \%$ & & $7,3 \%$ & 53981 & \\
\hline & & & & & & & & & & & & & & & & \\
\hline Tolal Ce & $71650 \quad 13.2 \%$ & 14157 & $2,6 \%$ & 76987 & $14,2 \%$ & 86517 & $12,3 \%$ & 66905 & $12,3 \%$ & 4273 & $0,8 \% \mathrm{p}$ & 199604 & 42387 & 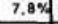 & 542480 & $100.0 \%$ \\
\hline Totsi Lo Caire & $210324 \quad 14,4 \%$ & 37701 & $2,6 \%$ & 222748 & $15.3 \%$ & 148558 & $10.0 \%$ & 187773 & $12.9 \%$ & 14309 & $1.0 \%$ & 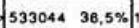 & 107390 & $7,4 \%$ & 1459847 & 100,09 \\
\hline
\end{tabular}

Source : CAPMAS, Recensement de la population 1976 - Gouvernorat du Caire, tableau 11

Tableau 6 : Répartition de la population active selon le statut dans la profession (6 ans au plus) - 1976

\begin{tabular}{|c|c|c|c|c|c|c|c|c|c|c|c|c|c|c|c|c|}
\hline \multirow[b]{2}{*}{ QISMS } & \multicolumn{16}{|c|}{ STATUT DANS LA PROFESSION } \\
\hline & \multicolumn{2}{|c|}{ Independants } & \multicolumn{2}{|c|}{ Employeurs } & \multicolumn{2}{|c|}{ Salaries } & \multicolumn{2}{|c|}{$\begin{array}{l}\text { Aides } \\
\text { tamillaux }\end{array}$} & \multicolumn{2}{|c|}{\begin{tabular}{|c|} 
Autres \\
travaillours \\
non renumberes
\end{tabular}} & \multicolumn{2}{|c|}{$\begin{array}{c}\text { Sans travail } ~ \\
\text { ayant deja } \\
\text { travallie }\end{array}$} & \multicolumn{2}{|c|}{$\begin{array}{l}\text { Sans travall I a la } \\
\text { recherche de leur } \\
\text { premier empioi }\end{array}$} & \multicolumn{2}{|c|}{ Total } \\
\hline $\begin{array}{l}\text { a). Wayyli } \\
\text { Manchiyyat Nasse }\end{array}$ & 4670 & $11,1 \times$ & 1313 & $3.1 x$ & 32340 & $76.9 \times$ & 87 & $0,2 x$ & - & $0.0 x$ & 236 & $0,6 \mathbf{x}$ & 3417 & 0,18 & 42049 & $100,0 \%$ \\
\hline & 2852 & $8.9 \times$ & 1980 & $6.2 x$ & 24269 & $75.7 x$ & 21 & $0,1 x$ & 10 & $0.0 \%$ & 176 & $0,5 \times$ & 2749 & $8.6 \%$ & 32057 & $100,0 x$ \\
\hline Bab a-Charia & 4770 & $13,5 \%$ & 1172 & $3,3 \%$ & 26929 & $76.4 \%$ & 98 & $0,3 \%$ & 2 & $0,0 \%$ & 138 & $0,4 \%$ & 2121 & $6.0 \%$ & 35228 & $100,0 \%$ \\
\hline at-Gamailyya & 8738 & $16,5 \%$ & 3964 & $7,5 \%$ & 36590 & $69,0 \%$ & 295 & $0,6 x$ & 17 & $0.0 x$ & 266 & $0.5 \%$ & 3180 & 6,0 & 53050 & $100,0 \%$ \\
\hline Darb al-Ahmar & 7431 & $15,6 \%$ & 1188 & $2.5 \%$ & 34186 & $71,5 \%$ & 1074 & $2,2 \%$ & 24 & & 381 & & 3497 & 7.3 & 47783 & $100,0 x$ \\
\hline Azbakiyya & 3187 & $14,7 \%$ & 1378 & 6.48 & 15168 & $70,6 \%$ & 53 & $0.2 x$ & 5 & & 233 & & 1494 & .7 .0 & 21496 & $100.0 \%$ \\
\hline Muski & 3393 & $18.2 x$ & 1298 & $7.0 \%$ & 12680 & $68,0 \%$ & 58 & $0.3 \%$ & 4 & $0,0 x$ & 99 & $0.5 \%$ & 1116 & 6.0 & 18628 & $100,0 \%$ \\
\hline Buileq & 11020 & $19.8 \%$ & 1749 & $3,1 x$ & 36315 & $69.0 x$ & 218 & $0,4 \%$ & 11 & $0,0 \%$ & 298 & $0.5 x$ & 3947 & 7.1 & 55558 & $100,0 \%$ \\
\hline Abdin & 3790 & $13,1 \mathrm{x}$ & 785 & $2,7 \%$ & 21825 & $75,7 x$ & 29 & $0,1 x$ & 3 & $0.0 \%$ & 206 & $0.7 \%$ & 2197 & 7.6 & 28835 & $100,0 \%$ \\
\hline $\begin{array}{l}\text { Oast al-Nil } \\
\text { Zamalek }\end{array}$ & 824 & $5,8 \times$ & 940 & $6.6 \%$ & 11624 & $81,8 x$ & 7 & $0.0 x$ & 2 & $0.0 x$ & 95 & $0,7 \pi$ & 714 & $5.0 \%$ & 14200 & $100.0 \%$ \\
\hline Sayyide Zaynab & 9374 & $11.6 \%$ & 2813 & $3.2 x$ & 61554 & $76.4 \%$ & 161 & $0.2 \%$ & 13 & $0.0 x$ & 412 & $0,5 \%$ & 6425 & $8.0 \%$ & 80552 & $100,0 \%$ \\
\hline Khalita & 7500 & $13,3 \times$ & 1422 & $2,5 \%$ & 43315 & $76,6 \%$ & 141 & $0,2 \%$ & 15 & $0,0 \%$ & 236 & $0,4 \%$ & 3929 & 6.96 & 56564 & $100,0 \%$ \\
\hline Masr al-Ouadima & 10353 & $12.7 \%$ & 2002 & $2,4 \%$ & 63132 & $77.3 x$ & 252 & $0.3 \%$ & 51 & $0,1 \%$ & 548 & $0,7 \%$ & 5384 & $6,6 \%$ & 81722 & $100,0 \%$ \\
\hline Total Centre ville & 77888 & $13.7 \%$ & 21804 & $3.8 \%$ & 421907 & $74,3 \%$ & 2472 & $0.4 \%$ & 163 & $0,0 \%$ & 3324 & $0.8 \%$ & 40170 & $7.1 \%$ & 567728 & $100,0 \%$ \\
\hline Total Le Caire & 181140 & $11,9 \%$ & 41464 & $2.7 \%$ & 1178055 & $77.1 \%$ & 4907 & $0,3 \mathbf{x}$ & 456 & $0.0 \%$ & 8176 & $0.5 x$ & 113407 & $7.4 \%$ & 1527614 & $100,0 \%$ \\
\hline
\end{tabular}

Source CAPMAS. Recensement de la population 1976 - Gouvernorat du Caire, tableau 13 Tableau 7 : Réparation de la population active par catégorie socio-professionnelle en 1976 (en \%) 


\begin{tabular}{|c|c|c|c|c|c|c|}
\hline \multirow{2}{*}{ QISMS } & \multicolumn{3}{|c|}{ Cots blancs } & \multirow{2}{*}{ Ouvriers } & \multirow{2}{*}{$\begin{array}{c}\text { Indépendants } \\
\& \text { Aides } \\
\text { familiaux }\end{array}$} & \multirow{2}{*}{ Salariẹs } \\
\hline & Total & & & & & \\
\hline $\begin{array}{l}\text { al-Wayti } \\
\text { Manchiyyat Nasser }\end{array}$ & 39.3 & 22,2 & 17.1 & 51,3 & 11,3 & 76,9 \\
\hline Zahir & 46.5 & 26,9 & 19.6 & 43,7 & 9,0 & 75.7 \\
\hline Bab al-Charia & 23,5 & 10,5 & 13,0 & 70,1 & 13.8 & 76,4 \\
\hline al-Gamaliyya & 15,1 & 6.1 & 9,0 & 78,3 & 17.1 & 69,0 \\
\hline Darb al-Ahmar & 22,6 & 10,3 & 12,3 & 65.9 & 17,8 & 71,5 \\
\hline Azbakiyya & 33,4 & 19,1 & 14,3 & 55,5 & 15.0 & 70,6 \\
\hline Muski & 21,4 & 10.9 & 10,5 & 71,1 & 18,5 & 68,0 \\
\hline Bulaq & 16,4 & 6,9 & 9,5 & 76,2 & 20.2 & 69,0 \\
\hline 'Abdin & 40,1 & 21.8 & 18.3 & 49,9 & 13.2 & 75.7 \\
\hline $\begin{array}{l}\text { Qasr al-Nit } \\
\text { Zamalek }\end{array}$ & 54,0 & 42,5 & 11,5 & 36,3 & 5.9 & 81,8 \\
\hline Sayyida Zaynab & 37,0 & 18,3 & 18.7 & 53,5 & 11,8 & 76,1 \\
\hline Khalifa & 25,0 & 11,2 & 13,8 & 67,4 & 13.5 & 76.6 \\
\hline Masr al-Qadima & 33.9 & 19.9 & 14.0 & 58,4 & 13,0 & 77,3 \\
\hline Total Centre Ville & 30,0 & 15,8 & 14,2 & 61.4 & 14.2 & 74,3 \\
\hline Total Le Caire & 32,3 & 17.0 & 15,3 & 59,4 & 12,2 & 77.1 \\
\hline
\end{tabular}

Sources : Tableaux 5 et 6

Tableau 8 : Matrice lieu d'habitation / lieu de travail -1976

\begin{tabular}{|c|c|c|c|c|c|c|c|c|c|c|c|c|}
\hline \multirow{3}{*}{$\begin{array}{l}\text { Lleu, } \\
\text { d'habltation } \\
\begin{array}{l}\text { al-Wayll } \\
\text { Manch. Nasser }\end{array}\end{array}$} & \multicolumn{12}{|c|}{ Lleu de travall } \\
\hline & \multicolumn{2}{|c|}{$\begin{array}{l}\text { A I'Interieur } \\
\text { du Cism }\end{array}$} & \multicolumn{2}{|c|}{$\begin{array}{l}\text { Centre des } \\
\text { atfaires } \\
\text { (CBD) }\end{array}$} & \multicolumn{2}{|c|}{$\begin{array}{c}\text { Centre } \\
\text { vilite }\end{array}$} & \multicolumn{2}{|c|}{$\begin{array}{l}\text { Hors centre ville } \\
\text { A I'Intérieur } \\
\text { du Gouvernorat }\end{array}$} & \multicolumn{2}{|c|}{ Indéterminess } & \multicolumn{2}{|c|}{ Total } \\
\hline & 19056 & $32.0 \%$ & 5008 & $8,4 \%$ & 28221 & $47,4 \%$ & 6551 & $11,0 \%$ & 673 & $1.1 \%$ & 59509 & $100,0 \%$ \\
\hline Zahlr & 9490 & $22,3 \%$ & 6157 & $14.5 \%$ & 22412 & $52,7 \%$ & 4321 & $10,2 \%$ & 176 & $0,4 \%$ & 42556 & $100,0 \%$ \\
\hline Bab al-Charla & 15356 & $30,1 \%$ & 4302 & $8,4 \%$ & 26990 & $53,0 \%$ & 3775 & $7.4 \%$ & 546 & $1,1 \%$ & 50969 & $100,0 \%$ \\
\hline al-Gamallyya & 22853 & $31.2 \%$ & 3887 & $5,3 \%$ & 35673 & $48,7 \%$ & 4151 & $5.7 \%$ & 6717 & $9,2 \%$ & 73281 & $100,0 \%$ \\
\hline Darb al-Ahmar & 24085 & $33,9 \%$ & 5105 & $7.2 \%$ & 37493 & $52,7 \%$ & 4092 & $5,8 \%$ & 321 & $0.5 \%$ & 71096 & $100,0 \%$ \\
\hline Azbakiyya & 9696 & $25,7 \%$ & 11582 & $30,7 \%$ & 13803 & $36,5 \%$ & 1933 & $5,1 \%$ & 754 & $2,0 \%$ & 37768 & $100,0 \%$ \\
\hline Muskl & 9430 & $24.2 \%$ & 2454 & $6,3 \%$ & 14367 & $36,8 \%$ & 12650 & $32,4 \%$ & 101 & $0,3 \%$ & 39002 & $100,0 \%$ \\
\hline Bulaq & 27944 & $33.7 \%$ & 7290 & $8.8 \%$ & 39322 & $47,5 \%$ & 6207 & $7,5 \%$ & 2100 & $2,5 \%$ & 82863 & $100,0 \%$ \\
\hline Abdin & 13273 & $24.3 \%$ & 17179 & $31,5 \%$ & 21244 & $38,9 \%$ & 2756 & $5,0 \%$ & 131 & $0.2 \%$ & 54583 & $100.0 \%$ \\
\hline $\begin{array}{l}\text { Qast al-NII } \\
\text { Zamalek }\end{array}$ & 8205 & $28.3 \%$. & 9281 & $32,0 \%$. & 10500 & $36.2 \%$ & 777 & $2.7 \%$ & 209 & $0.7 \%$ & 28972 & $100,0 \%$ \\
\hline Sayyida Zaynab & 32749 & $28,8 \%$ & 13624 & $12,0 \%$ & 55818 & $49,0 \%$ & 9180 & $8,1 \%$ & 2492 & $2,2 \%$ & 113863 & $100,0 \%$ \\
\hline Khalifa. & 21349 & $27,8 \%$ & 6313 & $8,2 \%$ & 39550 & $51,5 \%$ & 6339 & $8,3 \%$ & 3184 & $4,1 \%$ & 76735 & $100,0 \%$ \\
\hline Masr al-Oadima & 37930 & $32,5 \%$ & 10030 & $8,6 \%$ & 57505 & $49,3 \%$ & 9240 & $7,9 \%$ & 2050 & $1,8 \%$ & 116755 & $100.0 \%$ \\
\hline Total Centre ville & 251416 & $29,6 \%$ & 102212 & $12,1 \%$ & 402898 & $47,5 \%$ & 71972 & $8,5 \%$ & 19454 & $2,3 \%$ & 847952 & $100.0 \%$ \\
\hline Total Le Caire & 675886 & $.30 .7 \%$ & 196234 & $8.9 \%$ & 612813 & $27,8 \%$ & 682835 & $31,0 \%$ & 35800 & $1,6 \%$ & 2203568 & $100,0 \%$ \\
\hline
\end{tabular}

Source : CAPMAS, Recensement de la population 1976.

Tableau 9 : Matrice Lieu de travail / Lieu d'habitation 


\begin{tabular}{|c|c|c|c|c|c|c|c|c|c|c|}
\hline \multirow{2}{*}{$\begin{array}{l}\text { Lleu de } \\
\text { travail }\end{array}$} & \multicolumn{10}{|c|}{ Lleu d'habitation } \\
\hline & \multicolumn{2}{|c|}{$\begin{array}{l}\text { A l'intérieur } \\
\text { du qism }\end{array}$} & \multicolumn{2}{|c|}{ C.B.D } & \multicolumn{2}{|c|}{ centre ville } & \multicolumn{2}{|c|}{$\begin{array}{c}\text { A Plintérieur } \\
\text { du Gouvernorat } \\
\& \text { hors centre ville } \\
\end{array}$} & \multicolumn{2}{|c|}{ Total } \\
\hline Azbakiyya & 9696 & $16 \%$ & 11055 & $18 \%$ & 28035 & $46 \%$ & 33016 & $54 \%$ & 61051 & $10 \%$ \\
\hline ai-Gamaliyya & 22853 & $59 \%$ & 503 & $1 \%$ & 30856 & $79 \%$ & 8107 & $21 \%$ & 38963 & $6 \%$ \\
\hline Khalifa & 21349 & $77 \%$ & 181 & $1 \%$ & 24312 & $88 \%$ & 3298 & $12 \%$ & 27610 & $5 \%$ \\
\hline Darb al-Ahmar & 24085 & $71 \%$ & 502 & $1 \%$ & 30227 & $89 \%$ & 3657 & $11 \%$ & 33884 & $6 \%$ \\
\hline Sayyida Zaynab & 32749 & $55 \%$ & 1611 & $3 \%$ & 46126 & $78 \%$ & 13318 & $22 \%$ & 59444 & $10 \%$ \\
\hline Zahir & 9490 & $45 \%$ & 352 & $2 \%$ & 13560 & $64 \%$ & 7657 & $36 \%$ & 21217 & $3 \%$ \\
\hline Muski & 9430. & $22 \%$ & 1386 & $3 \%$ & 25637 & $60 \%$ & 16963 & $40 \%$ & 42600 & $7 \%$ \\
\hline at-Wayli & 19056 & $26 \%$ & 1244 & $2 \%$ & 34224 & $46 \%$ & 40282 & $54 \%$ & 74506 & $12 \%$ \\
\hline Bab al-Charia & 15356 & $59 \%$ & 282 & $1 \%$ & 20517 & $79 \%$ & 5331 & $21 \%$ & 25848 & $4 \%$ \\
\hline Bulaq & 27944 & $63 \%$ & 1059 & $2 \%$ & 33158 & $75 \%$ & 11336 & $25 \%$ & 44494 & $7 \%$ \\
\hline Abdin & 13273 & $27 \%$ & 14504 & $30 \%$ & 31220 & $64 \%$ & 17722 & $36 \%$ & 48942 & $8 \%$ \\
\hline Casr al-Nil & 8205 & $10 \%$ & 12483 & $14 \%$ & 42957 & $50 \%$ & 43284 & $50 \%$ & 86241 & $14 \%$ \\
\hline Masr al-Qadima & 37930 & $79 \%$. & 385 & $1 \%$ & 42069 & $88 \%$ & 5944 & $12 \%$ & 48013 & $8 \%$ \\
\hline TOTAL & 251416 & $41 \%$ & 45547 & $7 \%$ & 402898 & $66 \%$ & 209915 & $34 \%$ & 612813 & $100 \%$ \\
\hline
\end{tabular}

Tableau 10 : Répartition des établissements et des emplois d'après les recensements d'établissements de 1972, 1976 et 1986

\begin{tabular}{|c|c|c|c|c|c|c|c|c|c|}
\hline \multirow[b]{2}{*}{ Qisms } & \multicolumn{3}{|c|}{1972} & \multicolumn{3}{|c|}{1976} & \multicolumn{3}{|c|}{1986} \\
\hline & $\begin{array}{l}\text { Nombre } \\
\text { d'ètablis. } \\
\text { sements }\end{array}$ & $\begin{array}{l}\text { Nombre } \\
\text { ơemplois }\end{array}$ & $\begin{array}{l}\text { Taille } \\
\text { moyerne }\end{array}$ & $\begin{array}{c}\text { Nombre } \\
\text { d'êtablis. } \\
\text { sements }\end{array}$ & $\begin{array}{c}\text { Nombre } \\
\text { d'emplois }\end{array}$ & $\begin{array}{c}\text { Taille } \\
\text { mojeme }\end{array}$ & $\begin{array}{l}\text { Nombre } \\
\text { d'établis. } \\
\text { sements }\end{array}$ & $\begin{array}{l}\text { Nombre } \\
\text { d'emplois }\end{array}$ & $\begin{array}{l}\text { Taille } \\
\text { moyeme }\end{array}$ \\
\hline al-Wayli & 3813 & 17313 & 4,5 & 4352 & 19868 & 4,6 & 4565 & 14395 & 3,2 \\
\hline Manchiyyat Nasser & & & & & & & 4346 & 7608 & 1.8 \\
\hline Zahir & -4160 & 13364 & 3,2 & 4630 & 15588 & 3,4 & 4538 & 12784 & 2,8 \\
\hline Bab al-Charia & 4081 & 11994 & 2.9 & 4626 & 12356 & 2,7 & 5377 & 13052 & 2,4 \\
\hline al-Gamaliyya & 9561 & 29808 & 3,1 & 12037 & 33677 & 2,8 & 12246 & 34199 & 2.8 \\
\hline Darto at-Ahmar & 6987 & 16988 & 2,4 & 9526 & 22525 & 2,4 & 8936 & 22989 & 2.6 \\
\hline Azbakiyya & 7793 & 40790 & 5,2 & 8474 & 44684 & 5.3 & 8083 & 40360 & 5,0 \\
\hline Muski & 7294 & 25349 & 3,5 & 8100 & 25277 & 3.1 & 9056 & 26955 & 3.0 \\
\hline Bulaq & 7314 & 26559 & 3,6 & 7977 & 29805 & 3.7 & 8058 & 32023 & 4,0 \\
\hline Abdin & 10886 & 77612 & 7,1 & 11855 & 84024 & 7,1 & 11522 & 58856 & 5.1 \\
\hline Qasr al-Nil & 4060 & 44475 & 11,0 & 4007 & 40095 & 10,0 & 3656 & 40341 & 11,0 \\
\hline Zamalek & & & & & & 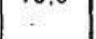 & 1736 & 14509 & 8,4 \\
\hline Sayyida Zaynab & 7921 & 21638 & 2,7 & 9042 & 24842 & 2.7 & 9272 & 20648 & 2,2 \\
\hline Khalita & 4218 & 9822 & 2,3 & 5006 & 11378 & 2,3 & 5500 & 13194 & 2,4 \\
\hline Masral-Oadima & 4584 & 31002 & 6,8 & 4307 & 22569 & 5,2 & 7978 & 24606 & 3.1 \\
\hline Total Centre Ville & 82672 & 366714 & 4,4 & 93939 & 386688 & 4,1 & 104869 & 376519 & 3.6 \\
\hline Total Le Caire & 141967 & 640654 & 4.5 & 168881 & 713518 & 4,2 & 236371 & 841016 & 3,6 \\
\hline
\end{tabular}

Sources : CAPMAS, Recensements des établissements. Résultats détaillés 1976 - Le Caire, 1981

CAPMAS, Recensements de la population des logements et des établissements. 1986, résultats préliminaires, Le Caire, 1987.

Tableau 11 : Taux de croissance du nombre d'établissements et de l'emploi 1976-1986 


\begin{tabular}{|c|c|c|c|}
\hline \multirow{2}{*}{ QISMS } & \multicolumn{2}{|c|}{ Taux annuel de croissance } & \multirow{2}{*}{$\begin{array}{l}\text { Ecarls des } \\
\text { tailles moyennes }\end{array}$} \\
\hline & Etablissements & Emplois & \\
\hline $\begin{array}{l}\text { al-Wayli } \\
\text { Manchiyyat Nasser } \\
\text { Zahir } \\
\text { Bab al-Charia } \\
\text { al-Garnaliyya } \\
\text { Darb ał-Ahmar } \\
\text { Azbakiyya } \\
\text { Muski } \\
\text { Bulaq } \\
\text { 'Abdin } \\
\text { Qasr al-Nil } \\
\text { Zamalek } \\
\text { Sayyida Zaynab } \\
\text { Khalifa } \\
\text { Masr al-Qadima }\end{array}$ & $\begin{array}{r}4,9 \\
-2,0 \\
16,2 \\
1,7 \\
-6,2 \\
-4,6 \\
11,8 \\
1,0 \\
-2,8 \\
34,6 \\
2,5 \\
9,9 \\
85,2\end{array}$ & $\begin{array}{r}-27,5 \\
-18,5 \\
5,6 \\
0,2 \\
0,2 \\
-9,7 \\
6.6 \\
7,4 \\
-29,9 \\
36,8 \\
\\
-16.9 \\
16.0 \\
9,0\end{array}$ & $\begin{array}{r}-1,41 \\
-0,54 \\
-0,24 \\
-0,01 \\
0,21 \\
-0,28 \\
-0,14 \\
0,23 \\
-1,98 \\
0,19 \\
-0,52 \\
0,13 \\
-2,16\end{array}$ \\
\hline Tolal Centre Ville & 11.6 & -2.6 & $-0,53$ \\
\hline Tolal Le Caire & 40,0 & 17,9 & $\cdot 0.66$ \\
\hline
\end{tabular}

Source : Tableau 10

Tableau 12 : Nombre d'établissements par habitant 1976-86

\begin{tabular}{|l|c|c|}
\hline \multicolumn{1}{|c|}{ QISMS } & $\begin{array}{c}\text { Nombre d'établissements } \\
\text { / Population } \\
1976\end{array}$ & $\begin{array}{c}\text { Nombre d'établissements } \\
\text { / Population } \\
1986\end{array}$ \\
\hline at-Wayli & 0.03 & 0.04 \\
Manchiyyat Nasser & 0.04 & 0.03 \\
Zahir & 0.04 & 0.05 \\
Bab at-Charia & 0.07 & 0.07 \\
al-Gamaliyya & 0.07 & 0.14 \\
Darb al-Ahmar & 0.14 & 0.08 \\
Azbakiyya & 0.14 & 0.18 \\
Muski & 0.04 & 0.21 \\
Bulaq & 0.14 & 0.07 \\
Abdin & 0.1 & 0.18 \\
Qasr al-Nit & 0.04 & 0.08 \\
Zamalek & 0.03 & 0.21 \\
Sayyida Zaynab & 0.02 & 0.05 \\
Khalifa & 0.05 & 0.03 \\
Masr al-Qadima & 0.03 & 0.03 \\
\hline Tolal Centre Ville & & 0.07 \\
\hline Total Le Caire & & 0.04 \\
\hline
\end{tabular}

Source : Tableaux 1 et 10

Tableau 13 : Répartition des emplois par qism et par branche d'activité économique, 1976 


\begin{tabular}{|c|c|c|c|c|c|c|c|c|c|c|c|c|c|c|c|c|}
\hline \multirow{3}{*}{ 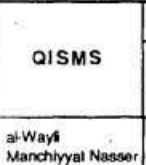 } & \multicolumn{16}{|c|}{ BRANCHES D'ACTIVITE ECONOMIOUE } \\
\hline & \multicolumn{2}{|c|}{\begin{tabular}{|c|} 
Industries \\
Manutacturiéres
\end{tabular}} & \multicolumn{2}{|c|}{ Construction } & \multicolumn{2}{|c|}{ Commerce } & \multicolumn{2}{|c|}{$\begin{array}{c}\text { Restaurants } \\
\text { \& Hotels }\end{array}$} & \multicolumn{2}{|c|}{ Transport } & \multicolumn{2}{|c|}{ Banques } & \multicolumn{2}{|c|}{\begin{tabular}{|l|} 
Administratits, \\
Servicos sociaux \\
et personnels (")
\end{tabular}} & \multicolumn{2}{|c|}{ TOTAL } \\
\hline & 8228 & $41.4 \%$ & 68 & $0,3 \%$ & 3882 & $19.6 \%$ & 701 & $3.5 \%$ & 4010. & $20.2 \%$ & 153 & $0,8 \%$ & 2814 & $14.2 \%$ & 19856 & $100,0 \%$ \\
\hline & 5826 & $35.9 \%$ & 125 & $0,8 \%$ & 3755 & $23.2 \%$ & 575 & $3.5 \%$ & 75 & $0.5 \%$ & 180 & $1.1 \%$ & 5681 & $35,0 \%$ & 16217 & $100,0 \%$ \\
\hline Bab at & 6539 & $53,2 \%$ & & & 3468 & 28,2 & & & 49 & & & & 1527 & $12,4 \%$ & 12286 & $100.0 \%$ \\
\hline & 16295 & $50,9 \%$ & 8 & $0,3 \%$ & $\begin{array}{r}34681 \\
13241\end{array}$ & \begin{tabular}{|l}
28,2 \\
41,3
\end{tabular} & 1566 & $\begin{array}{l}3,8 \% \\
4,9 \%\end{array}$ & 224 & & $\begin{array}{l}610 \\
610\end{array}$ & & $\begin{array}{r}301 \\
0\end{array}$ & $0,0 \%$ & 32031 & $100.0 \%$ \\
\hline Datha & 10366 & $\begin{array}{l}36,96 \\
46,96\end{array}$ & ${ }_{64}^{\infty}$ & $0,3 \%$ & $\begin{array}{l}8753 \\
8751\end{array}$ & $\begin{array}{l}41,3 \\
39,6 \\
\end{array}$ & 882 & & 131 & & $\begin{array}{l}810 \\
153\end{array}$ & & 1746 & $7,9 \%$ & 22095 & $100,0 \%$ \\
\hline$A z$ & 11781 & $\begin{array}{l}46,6 \% \\
26,56\end{array}$ & $\begin{array}{r}644 \\
5643\end{array}$ & $12,7 \%$ & $\begin{array}{r}8733 \\
15332\end{array}$ & $\begin{array}{l}39,6 \\
34.6\end{array}$ & & & & & 2398 & & 5246 & $11,8 \%$ & $\begin{array}{l}44275 \\
\end{array}$ & $100,0 \%$ \\
\hline & 575 & $\begin{array}{l}25,6 \\
45,8\end{array}$ & $\begin{array}{l}543 \\
117\end{array}$ & $0.5 \%$ & $\begin{array}{r}13332 \\
9962\end{array}$ & $\begin{array}{l}34 \\
39\end{array}$ & & & & & 419 & & $\begin{array}{l}1412 \\
1412\end{array}$ & $5.6 \%$ & 25268 & $100.0 \%$ \\
\hline But & 13535 & 45,5 & & & $\begin{array}{l}93062 \\
9332\end{array}$ & 31 & & & & & 220 & $0.7 \%$ & & $9.4 \%$ & 29731 & $100.0 \%$ \\
\hline & & $20,2 \%$ & 4458 & $5,4 \%$ & 20614 & & & & & & 28715 & $34.9 \%$ & & $9.3 \%$ & 82315 & $100,0 \%$ \\
\hline Oasr : & 5694 & $16,9 \%$ & 4784 & $14,2 \%$ & $\begin{array}{l}20614 \\
10614\end{array}$ & $31,5 \%$ & 2471 & $7,3 \%$ & 2002 & $8,3 \%$ & $\mid \begin{array}{r}20181 \\
1821\end{array}$ & $5.7 \%$ & 5405 & $16,0 \%$ & 33691 & $100,0 \%$ \\
\hline Zamak & 10511 & & & & & & & & & & & & & & 24830 & $00 \%$ \\
\hline $\begin{array}{l}\text { Sayvid } \\
\text { Khalita }\end{array}$ & $\begin{array}{r}10511 \\
5213\end{array}$ & & & & $\begin{array}{l}716 \\
338\end{array}$ & & & & $\begin{array}{r}1149 \\
43\end{array}$ & & $\begin{array}{r}320 \\
86\end{array}$ & & & & $\begin{array}{l}248303 \\
11203\end{array}$ & \\
\hline Mass w odadma & 8476 & $36,9 \%$ & 1105 & $4,8 \%$ & 6111 & $26,6 \%$ & $\begin{array}{l}1379 \\
1379\end{array}$ & $6,0 \%$ & 1779 & $7.7 \%$ & 260 & $1,1 \%$ & 3869 & $16,8 \%$ & 22979 & $100.0 \%$ \\
\hline Total Centro Vilite & 130643 & $34.7 \%$ & 17728 & $4,7 \%$ & 115529 & $30,7 \%$ & 18520 & $4.9 \%$ & 14907 & $4.0 \%$ & 35579 & $9.4 \%$ & 43971 & $11.6 \%$ & 376777 & $100.0 \%$ \\
\hline Toal Le Caire & 301971 & $46.2 \%$ & 29736 & $4,6 \%$ & 130596 & $20.0 \%$ & 27286 & $4.2 \%$ & 35597 & $5,4 \%$ & 43910 & $.6,7 \%$ & 84328 & $12,9 \%$ & 653424 & $100,0 \%$ \\
\hline
\end{tabular}

Source : CAPMAS, Recensement des Établissements, 1976

(*) Hors Administration publique

Tableau 14 : Répartition des emplois manufacturiers par qism et par branche d'activité, 1976

\begin{tabular}{|c|c|c|c|c|c|c|c|c|c|c|c|c|c|c|c|c|}
\hline \multirow{3}{*}{$\begin{array}{c}\text { QISMS } \\
\text { atWay } \\
\text { Manchinyat Nasser }\end{array}$} & \multicolumn{16}{|c|}{ BRANCHES D'ACTIVITE ECONOMIQUE } \\
\hline & \multicolumn{2}{|c|}{$\begin{array}{c}\text { Industries } \\
\text { Manutacturières }\end{array}$} & \multicolumn{2}{|c|}{$\begin{array}{c}\text { Industries } \\
\text { Allmentaires }\end{array}$} & \multicolumn{2}{|c|}{$\begin{array}{c}\text { Texbile, } \\
\text { Habillement, } \\
\text { Chaussures }\end{array}$} & \multicolumn{2}{|c|}{$\begin{array}{c}\text { Bois et } \\
\text { Imprimerie }\end{array}$} & \multicolumn{2}{|c|}{$\begin{array}{l}\text { Industries } \\
\text { chimiques }\end{array}$} & \multicolumn{2}{|c|}{$\begin{array}{c}\text { Industriles non } \\
\text { métaliques }\end{array}$} & \multicolumn{2}{|c|}{$\begin{array}{l}\text { Industries } \\
\text { du métal }\end{array}$} & \multicolumn{2}{|c|}{ Autres } \\
\hline & 8228 & $100.0 \%$ & 872 & $10.6 \%$ & 1609 & $19.6 \%$ & 1000 & $122 \%$ & 126 & $15 \%$ & 610 & $75 \%$ & 2909 & $35.4 \%$ & 1093 & $13.3 \%$ \\
\hline & 5826 & $100.0 \%$ & 743 & $12.8 \%$ & 1259 & $21.6 \%$ & 2230 & $38.3 \%$ & 252 & $4.3 \%$ & 108 & $1.9 \%$ & 1224 & $21.0 \%$ & 10 & $0.2 \%$ \\
\hline & & & 1404 & 21.5 & & & & & 110 & $18 \%$ & 114 & & 1078 & 16.5 & 141 & $22 \%$ \\
\hline & & 100. & 1012 & & & & 56 & & 332 & $\%$ & 238 & $1.5 \%$ & 4040 & $24.8 \%$ & 1940 & $11.8 \%$ \\
\hline & & 100. & 845 & & & & & & 35 & $\%$ & 500 & & 1317 & 12. & 231 & $22 \%$ \\
\hline & & & 1405 & 11.8 & & & & & 668 & & 8 & & & 19. & 06 & $0.8 \%$ \\
\hline & & & & & 30 & 56. & & & & & & & & 2. & 155 & $13 \%$ \\
\hline But & & & 1032 & & 73 & & & & 13 & & 427 & & 3007 & $28.3 \%$ & 1 & $0.0 \%$ \\
\hline & 166 & 100 & 2178 & $13.1 \%$ & 7645 & $46.0 \%$ & 2745 & $16.5 \%$ & 802 & $4.8 \%$ & 191 & $12 \%$ & 2896 & $17.4 \%$ & 147 & $0.8 \%$ \\
\hline $\begin{array}{l}\text { Casr at-Nil } \\
\text { Zametek }\end{array}$ & & $100.0 \%$ & 1240 & $218 \%$ & 1704 & $29.9 \%$ & 1268 & $22.3 \%$ & $\pi 1$ & $13.5 \%$ & 70 & $12 \%$ & 627 & $11.0 \%$ & 14 & 0276 \\
\hline Saypida Zaynab & 10511 & $100.0 \%$ & 3411 & $32.5 \%$ & 1767 & $16.8 x$ & 3650 & $34.7 \%$ & 86 & $0.8 \%$ & 107 & $10 \%$ & 1455 & 13. & 36 & $0.3 \%$ \\
\hline & & $100.0 \%$ & 606 & & & $44.7 \%$ & & 13 & 35 & & 398 & $73 x$ & 1080 & 20. & $\pi$ & $15 \%$ \\
\hline Mase \&-Oadma & 8476 & $100.0 \%$ & 1260 & $14.9 \%$ & 3878 & $45.8 \%$ & 964 & $11.3 \%$ & 474 & $5.6 \%$ & 1337 & $15.8 \%$ & 547 & $6.5 \%$ & 26 & $0.3 \%$ \\
\hline Total Contre Valie & 130643 & $100.0 \%$ & 16732 & $12.8 \%$ & 43778 & $33.5 \%$ & 32519 & $24.9 \%$ & 4400 & $3.4 \%$ & 4785 & $3.7 x$ & 24452 & $18.7 \%$ & 3977 & $3.0 \%$ \\
\hline Total Le Cairo & 301971 & $100.0 \%$ & 29671 & $9.8 \%$ & 89178 & $29.5 \%$ & 30035 & $10.2 \%$ & 27662 & $9.2 \%$ & 16488 & $5.5 \%$ & 66767 & $22.1 \%$ & 4028 & $1.6 \%$ \\
\hline
\end{tabular}

Source : CAPMAS, Recensement des Établissements. 1976

Tableau 15 : Spécialisations manufacturières des qisms du centre du Caire

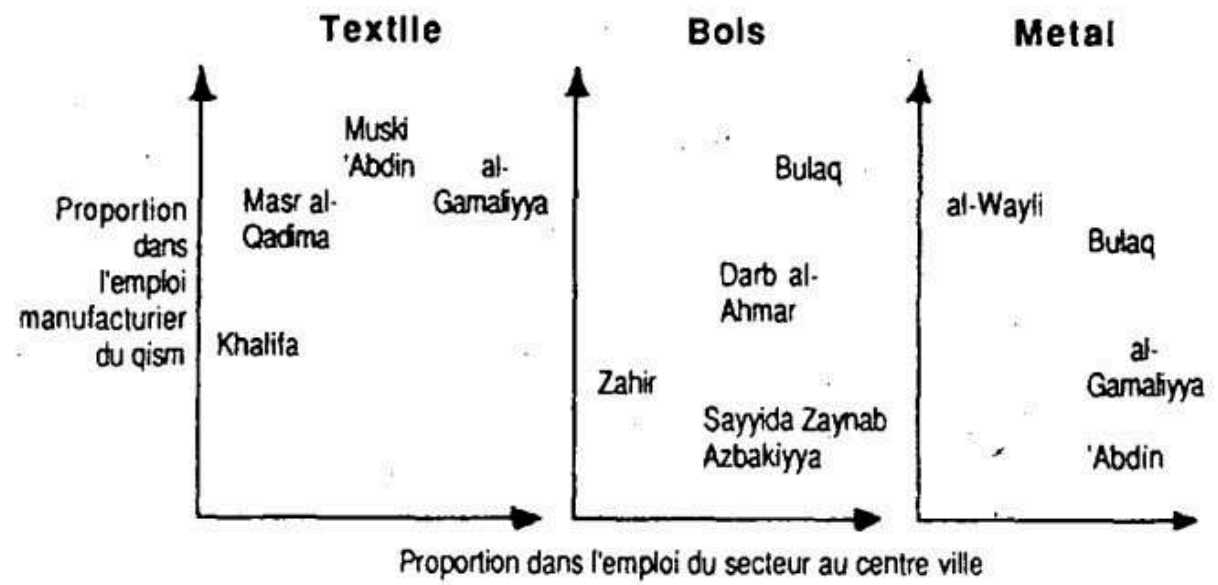

Tableau 16 : Taille des établissements par qism et par branche d'activité économique 1976 


\begin{tabular}{|c|c|c|c|c|c|c|c|c|}
\hline \multirow[b]{2}{*}{ QISMS } & \multicolumn{8}{|c|}{ BRANCHES } \\
\hline & $\begin{array}{c}\text { industries } \\
\text { manutacturitres }\end{array}$ & Construction & Commerce & $\mid \begin{array}{c}\text { Reslaurants } \\
\text { \& hotets }\end{array}$ & Transponts & Banques & $\begin{array}{l}\text { Services } \\
\text { administratis sociaix } \\
\text { al personnetel') }\end{array}$ & Total \\
\hline 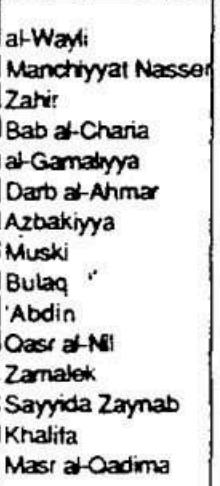 & $\begin{array}{l}9.3 \\
3.79 \\
3.8 \\
4.06 \\
3.66 \\
5.99 \\
4.1 \\
8.1 \\
4.86 \\
9.23 \\
1.33 \\
3.66 \\
5.41\end{array}$ & $\begin{array}{r}1.94 \\
1.98 \\
1.54 \\
1.56 \\
1.23 \\
29.09 \\
1.98 \\
7.46 \\
14.06 \\
32.11 \\
\\
2.24 \\
3.73 \\
16.49\end{array}$ & $\begin{array}{l}1.9 \\
2 \\
1,9 \\
2.1 \\
1.9 \\
4.2 \\
2,6 \\
2.1 \\
5.1 \\
6.7 \\
1.7 \\
1.5 \\
1.9\end{array}$ & $\begin{array}{r}2.19 \\
2.41 \\
2.19 \\
2.41 \\
2.11 \\
5.27 \\
3.31 \\
2.38 \\
4.81 \\
11.39 \\
\\
2.29 \\
1.94 \\
2.92\end{array}$ & $\begin{array}{c}455.56 \\
\\
3.75 \\
2.88 \\
2.38 \\
3.12 \\
8.76 \\
2.64 \\
25,2 \\
11.88 \\
22.78 \\
\\
57.45 \\
1.87 \\
53.91\end{array}$ & $\begin{array}{l}2,9 \\
4,6 \\
4 \\
6,9 \\
5,5 \\
4,9 \\
3.2 \\
5,2 \\
28 \\
6,4 \\
3,8 \\
2.3 \\
5.1\end{array}$ & $\begin{array}{l}2.99 \\
5.03 \\
2.08 \\
1.73 \\
1.93 \\
3.67 \\
2.06 \\
2.47 \\
3.23 \\
5.42 \\
2.15 \\
1.89 \\
2.91-\end{array}$ & $\begin{array}{r}4,6 \\
3,4 \\
2,7 \\
2,8 \\
2,4 \\
5.3 \\
3,1 \\
3.7 \\
7,1 \\
10 \\
\\
2,8 \\
2.3 \\
3,4\end{array}$ \\
\hline Total Contre Vilie & 4,03 & 13.49 & 2.7 & 3.06 & 20.39 & 15 & 2.97 & 4 \\
\hline Total Le Caire & 7.51 & 13.97 & 1.6 & $2 . \pi$ & 27.26 & 15 & 2.73 & 4.2 \\
\hline
\end{tabular}

$\left(^{*}\right)$ hors Administration publique

Source : CAPMAS. Recensement des établissements 1976.

Tableau 17 : Unités d'habitation utilisées pour l'exercice d'activités économiques selon le recensement de population, 1976 - 1986

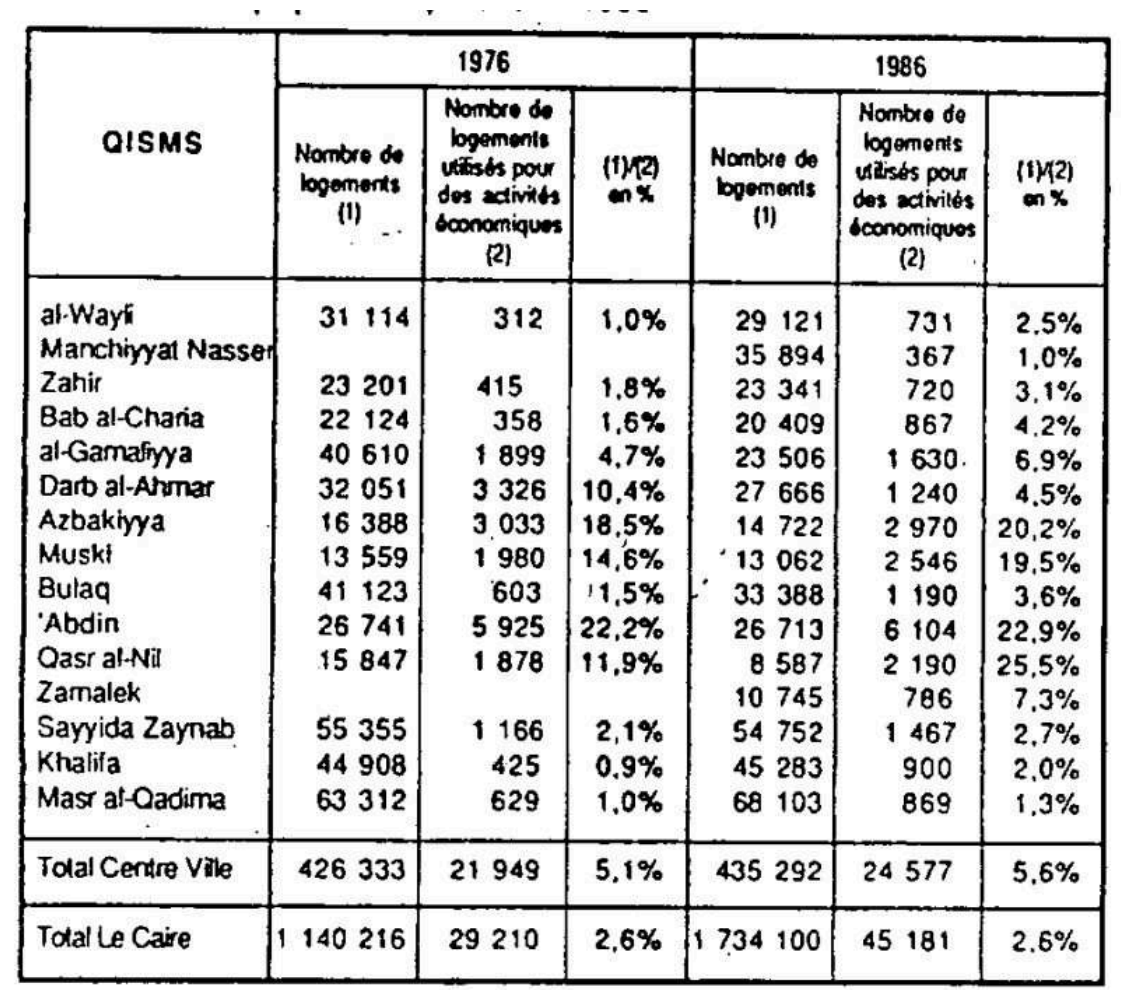

Usage mixte inclus

Source : CAPMAS, Recensements de population 1976, 1986

Tableau 18 : Classement des qisms selon la proportion d'habitation utilisées pour l'exercice d'activités 1976 - 1986 


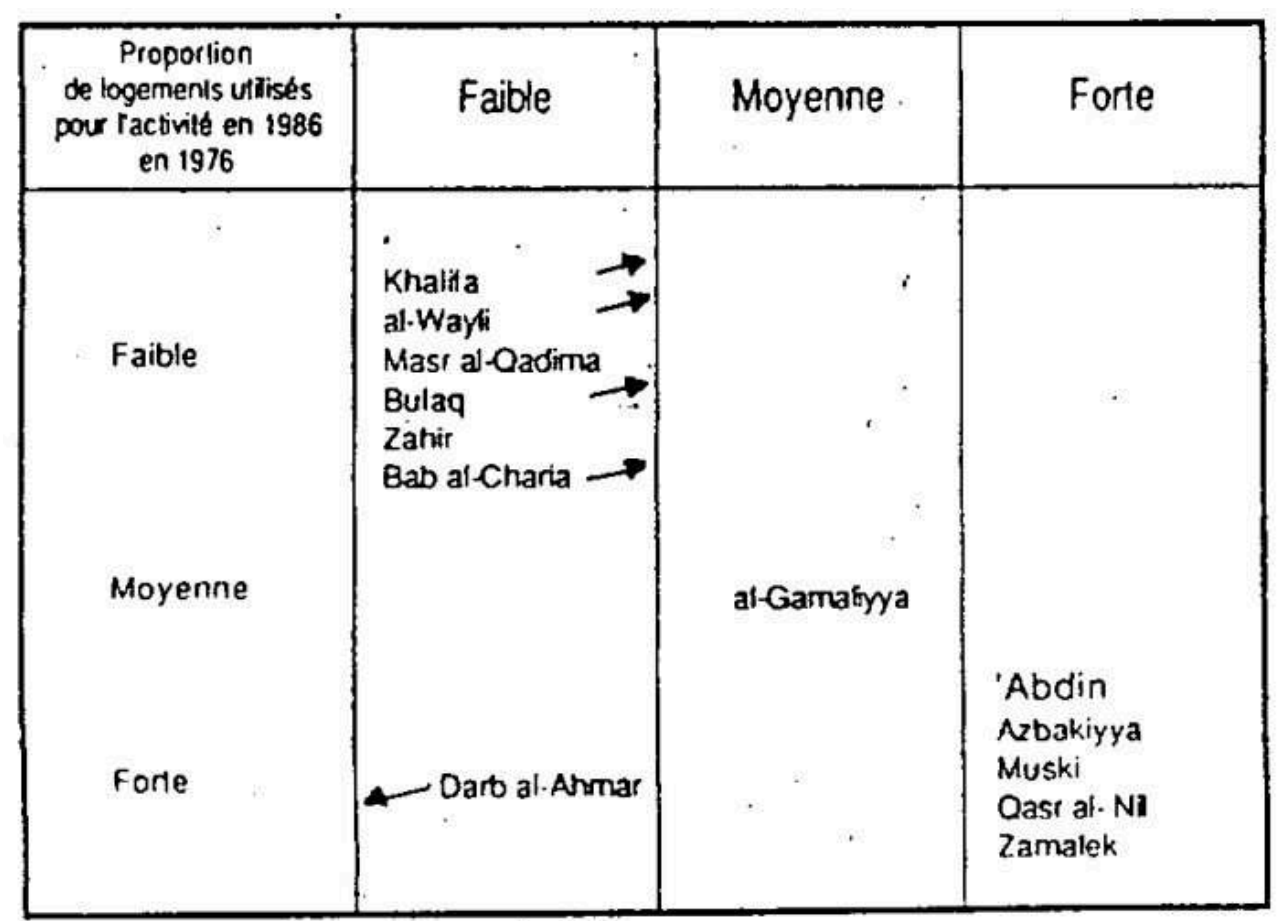

Tableau 19 : Nombre d'établissements cachés estimé à partir de la comparaison entre le recensement de population et le recensement d'établissements, 1976/1986

\begin{tabular}{|c|c|c|c|c|c|c|c|c|c|c|c|c|c|}
\hline \multirow[b]{3}{*}{ OISMS } & \multicolumn{7}{|c|}{1976} & \multicolumn{6}{|c|}{1986} \\
\hline & \multicolumn{3}{|c|}{$\begin{array}{c}\text { Fecensement } \\
\text { des établissements }\end{array}$} & \multirow{2}{*}{ 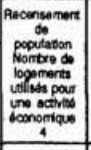 } & \multirow{2}{*}{ 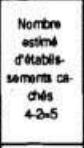 } & \multirow{2}{*}{ 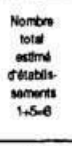 } & \multirow{2}{*}{ 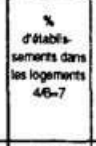 } & \multirow[b]{2}{*}{ 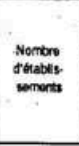 } & \multirow{2}{*}{ 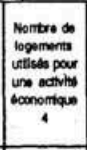 } & \multirow{2}{*}{ 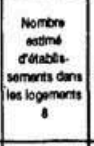 } & \multirow{2}{*}{ 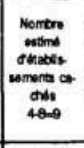 } & \multirow{2}{*}{ 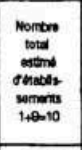 } & \multirow{2}{*}{ 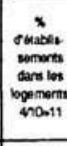 } \\
\hline & 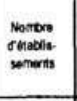 & 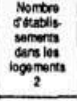 & $2 n \infty$ & & & & & & & & & & \\
\hline $\begin{array}{l}\text { atWayd } \\
\text { Manchilyyat Nasser }\end{array}$ & 4352 & 267 & $6.1 \%$ & 312 & 45 & 4397 & $7.1 \%$ & $\begin{array}{l}4565 \\
4346\end{array}$ & $\begin{array}{l}731 \\
367\end{array}$ & $\begin{array}{l}278 \\
265\end{array}$ & $\begin{array}{l}453 \\
102\end{array}$ & $\begin{array}{l}5018 \\
4448\end{array}$ & $\begin{array}{l}14.6 \% \\
8,3 \%\end{array}$ \\
\hline Zahit & 4630 & 446 & $9.6 \%$ & 415 & -31 & 4630 & $9,0 \%$ & & 720 & 436 & 284 & 4822 & $14.9 \%$ \\
\hline Bab atCharia & & 342 & & & 16 & & 7.7 & & 867 & 398 & 469 & 5846 & $14,8 \%$ \\
\hline a)-Camallyya & 12047 & 1685 & $14.0 \%$ & 1899 & 214 & 12261 & 15,5 & 12246 & 1630 & 1714 & $-\infty$ & 12246 & $13,3 \%$ \\
\hline Datb at-Armar & 9526 & 351 & $3.7 \%$ & 3326 & 2975 & 12501 & 26,6 & 8936 & 1240 & 331 & & & $12,6 \%$ \\
\hline Azbekivya & 8474 & 2740 & $32,3 \%$ & 3033 & 293 & 8767 & $34,6 \%$ & 808 & 29 & 26 & 359 & 8442 & $35.2 \%$ \\
\hline Musta & 8100 & 1138 & $14,0 \%$ & 1980 & 842 & 8942 & $22,1 \%$ & 905 & 2546 & 126 & 1278 & 10334 & $24,6 \%$ \\
\hline Buleq & 7977 & 546 & $6,8 \%$ & 603 & 57 & 8034 & $7,5 \%$ & 805 & 1190 & 54 & 642 & 8700 & $13.7 \%$ \\
\hline Abdin & 11855 & 4121 & $34.8 \%$ & 5925 & 1804 & 13659 & $43,4 \%$ & 11522 & 6104 & 40 & 209 & 13616 & $44,8 \%$ \\
\hline Casr at-Nil & 4007 & 1349 & $33,7 \%$ & 1878 & 529 & 4536 & $41,4 \%$ & 3656 & 2190 & 123 & 95 & 4614 & $47.5 \%$ \\
\hline Zamal & & & & & & & & & 786 & 58 & & 1937 & $40,6 \%$ \\
\hline Saypic & 9042 & & $8.2 \%$ & 1166 & 421 & 9463 & $12,3 \%$ & 9272 & 1467 & 76 & 707 & 9979 & $14.7 \%$ \\
\hline & 5006 & & 5,0 & 425 & 177 & 5183 & 8.2 & 550 & 900 & 27 & 63 & 6130 & $14,7 \%$ \\
\hline Masr ab Oadime & 4307 & 299 & $6,9 \%$ & 629 & 330 & 4637 & $13.6 \%$ & 7978 & 869 & 550 & 319 & 8297 & $10.5 \%$ \\
\hline Total Centre Vilie & 93939 & 14277 & $15,2 \%$ & 21949 & 7672 & 101611 & $21,6 \%$ & 104869 & 24577 & 15256 & 9321 & 114190 & $21,5 \%$ \\
\hline Total Le Carbe & 168881 & 18819 & $11,1 \%$ & 29210 & 10391 & 179272 & $16,3 \%$ & 236371 & 45181 & 26237 & 18944 & 255315 & $17.7 \%$ \\
\hline
\end{tabular}

Source : Tableaux $11 \& 13$ précédents.

Carte 1 : Le Caire 


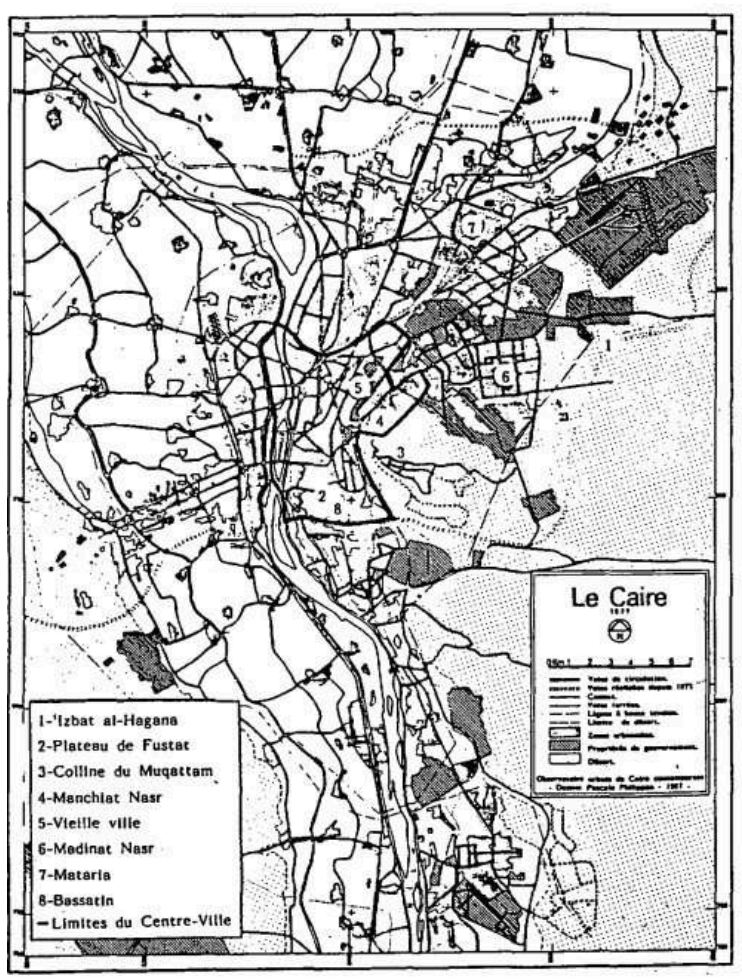

Carte2 : Les qism du centre ville du Caire

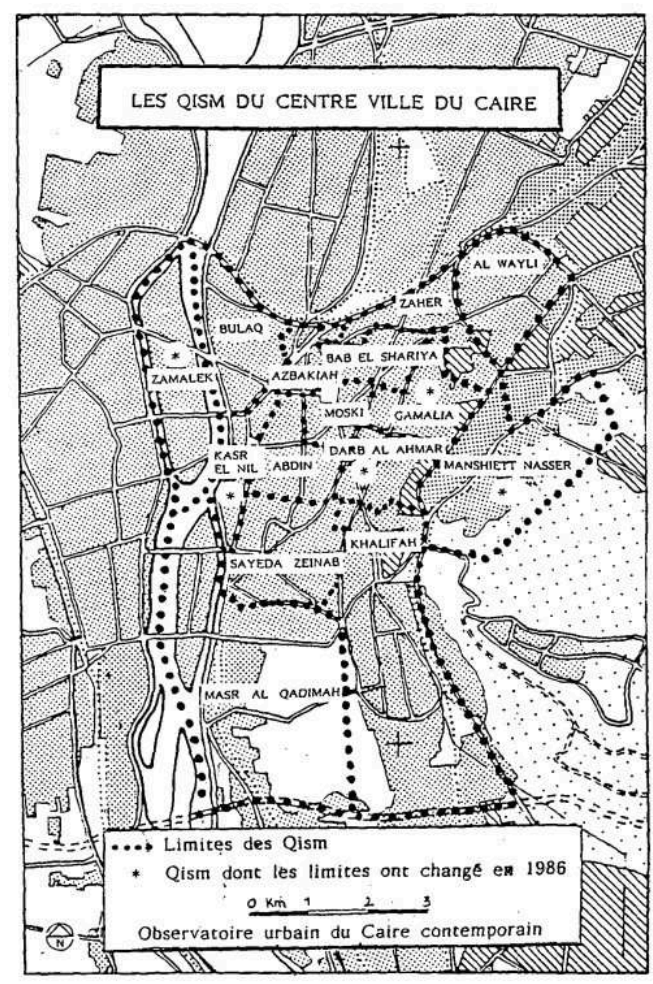

\section{INDEX}

Mots-clés : recensement, Le Caire, emploi 
AUTEUR

JACQUES CHARMES

ORSTOM/INSEE 